\title{
THE RICCI-BOURGUIGNON FLOW
}

\author{
GIOVANNI CATINO, LAURA CREMASCHI, ZINDINE DJADLI, CARLO MANTEGAZZA, \\ AND LORENZO MAZZIERI
}

\begin{abstract}
In this paper we present some results on a family of geometric flows introduced by J. P. Bourguignon in [3] that generalize the Ricci flow. For suitable values of the scalar parameter involved in these flows, we prove short time existence and provide curvature estimates. We also state some results on the associated solitons.
\end{abstract}

\section{INTRODUCTION}

In this paper we consider an $n$-dimensional, compact, smooth, Riemannian manifold $M$ (without boundary) whose metric $g=g(t)$ is evolving according to the flow equation

$$
\frac{\partial}{\partial t} g=-2 \operatorname{Ric}+2 \rho \mathrm{R} g=-2(\operatorname{Ric}-\rho \mathrm{R} g)
$$

where Ric is the Ricci tensor of the manifold, R its scalar curvature and $\rho$ is a real constant. This family of geometric flows contains, as a special case, the Ricci flow, setting $\rho=0$. Moreover, by a suitable rescaling in time, when $\rho$ is nonpositive, they can be seen as an interpolation between the Ricci flow and the Yamabe flow (see $[4,27,30]$, for instance), obtained as a limit when $\rho \rightarrow-\infty$.

It should be noticed that for special values of the constant $\rho$ the tensor Ric $-\rho \mathrm{R} g$ appearing on the right hand side of the evolution equation is of special interest, in particular,

- $\rho=1 / 2$, the Einstein tensor Ric $-\frac{\mathrm{R}}{2} g$,

- $\rho=1 / n$, the traceless Ricci tensor Ric $-\frac{\mathrm{R}}{n} g$, 
- $\rho=1 / 2(n-1)$, the Schouten tensor Ric $-\frac{\mathrm{R}}{2(n-1)} g$,

- $\rho=0$, the Ricci tensor Ric.

In dimension two, the first three tensors are zero hence the flow is static, and in higher dimension the values of $\rho$ are strictly ordered as above, in descending order.

Apart these special values of $\rho$, for which we will call the associated flows as the name of the corresponding tensor, in general we will refer to the evolution equation defined by the PDE system (1.1) as the Ricci-Bourguignon flow (or shortly RB flow).

The study of these flows was proposed by Jean-Pierre Bourguignon in [3, Question 3.24], building on some unpublished work of Lichnerowicz in the sixties and a paper of Aubin [1]. In 2003, Fischer [16] studied a conformal version of this problem where the scalar curvature is constrained along the flow. In 2011, Lu, Qing and Zheng [23] also proved some results on the conformal Ricci-Bourguignon flow.

We will see in the next section that when $\rho$ is larger than $1 / 2(n-1)$ the principal symbol of the operator in the right hand side of the second order quasilinear parabolic PDE (1.1) has negative eigenvalues, not allowing even a short time existence result for the flow for general initial data (manifold $M$ and initial metric $g_{0}$ ). On the contrary, the main task of Section 2 will be to show that for any $\rho<1 / 2(n-1)$, every initial compact Riemannian manifold $\left(M, g_{0}\right)$ has a unique smooth solution $g(t)$ solving the flow equation (1.1), with $g(0)=g_{0}$, at least in a positive time interval.

However, the problem of knowing whether the "critical" Schouten flow

$$
\left\{\begin{array}{l}
\frac{\partial}{\partial t} g=-2 \mathrm{Ric}+\frac{\mathrm{R}}{n-1} g \\
g(0)=g_{0}
\end{array}\right.
$$

when $\rho=1 / 2(n-1)$, admits or not a short time solution for general initial manifolds and metrics remains open, when $n \geq 3$.

We will see that if $\rho \leq 1 / 2(n-1)$, the principal symbol of the elliptic operator is nonnegative definite and it actually contains some zero eigenvalues due to the diffeomorphism invariance of the geometric flow. When $\rho<1 / 2(n-1)$, these zero eigenvalues are the only ones, all the others are indeed positive, hence, they can be dealt with (as it is customary by now) by means of the so-called DeTurck's trick [13,14]. In the case of the Schouten flow $\rho=1 / 2(n-1)$ instead, the principal symbol contains an extra zero eigenvalue besides the ones due to the diffeomorphism invariance, preventing this argument to be sufficient to conclude and to give a general short time existence result.

We mention that the presence of this extra zero eigenvalue should be expected, as the Cotton tensor, which is obtained from the Schouten tensor as follows

$$
\mathrm{C}_{i j k}=\nabla_{k} \mathrm{R}_{i j}-\nabla_{j} \mathrm{R}_{i k}=\nabla_{k} \mathrm{R}_{i j}-\nabla_{j} \mathrm{R}_{i k}-\frac{1}{2(n-1)}\left(\nabla_{k} \mathrm{R} g_{i j}-\nabla_{j} \mathrm{R} g_{i k}\right),
$$

satisfies the following invariance under the conformal change of metric $\widetilde{g}=e^{2 u} g$,

$$
e^{3 u} \widetilde{\mathrm{C}}_{i j k}=\mathrm{C}_{i j k}+(n-2) \mathrm{W}_{i j k l} \nabla^{l} u
$$


see [7, Equation 3.35]. Recently, Delay [12], following the work of Fischer and Marsden, gave some evidence on the fact that the DeTurck's trick should fail for the Schouten tensor.

In Section 3, we will compute the evolution equations for the curvature.

In Section 4, by means of the maximum principle, we derive, from the evolution of the curvature, some conditions on the curvature which are preserved by the RB flow. In particular, we show that the Hamilton-Ivey estimate

Acknowledgments. We want to thank Mauro Carfora for several suggestions and comments. The authors are partially supported by the GNAMPA project "Equazioni di evoluzione geometriche e strutture di tipo Einstein". Z.D. is partially supported by the grant GTO: ANR-12BS01-0004.

\subsection{Notation and preliminaries.}

The Riemann curvature operator of an $n$-dimensional Riemannian manifold $(M, g)$ is defined as in [17] by

$$
\operatorname{Riem}(X, Y) Z=\nabla_{Y} \nabla_{X} Z-\nabla_{X} \nabla_{Y} Z+\nabla_{[X, Y]} Z
$$

and we will denote with $d \mu_{g}$ the canonical volume measure associated to the metric $g$. In a local coordinate system the components of the $(3,1)$-Riemann curvature tensor are given by $\mathrm{R}_{i j k}^{l} \frac{\partial}{\partial x^{l}}=\operatorname{Riem}\left(\frac{\partial}{\partial x^{i}}, \frac{\partial}{\partial x^{j}}\right) \frac{\partial}{\partial x^{k}}$ and we denote by $\mathrm{R}_{i j k l}=g_{l m} \mathrm{R}_{i j k}^{m}$ its $(4,0)$-version. With this choice, for the sphere $\mathbb{S}^{n}$ we have $\operatorname{Riem}(v, w, v, w)=\mathrm{R}_{i j k l} v^{i} w^{j} v^{k} w^{l}>0$.

The Ricci tensor is obtained as the contraction $\mathrm{R}_{i k}=g^{j l} \mathrm{R}_{i j k l}$ and $\mathrm{R}=g^{i k} \mathrm{R}_{i k}$ will denote the scalar curvature.

The so-called Weyl tensor is then defined by the decomposition formula (see [17, Chapter 3, Section K]) of the Riemann tensor in dimension $n \geq 3$,

$$
\mathrm{W}_{i j k l}=\mathrm{R}_{i j k l}+\frac{\mathrm{R}}{(n-1)(n-2)}\left(g_{i k} g_{j l}-g_{i l} g_{j k}\right)-\frac{1}{n-2}\left(\mathrm{R}_{i k} g_{j l}-\mathrm{R}_{i l} g_{j k}+\mathrm{R}_{j l} g_{i k}-\mathrm{R}_{j k} g_{i l}\right) \text {. }
$$

The tensor $\mathrm{W}$ satisfies all the symmetries of the curvature tensor and all its traces with the metric are zero, as it can be easily seen from the above formula.

In dimension three, $\mathrm{W}$ is identically zero for every Riemannian manifold $(M, g)$, and it becomes relevant instead when $n \geq 4$ since it vanishes if and only if $(M, g)$ is locally conformally flat. This latter condition means that around every point $p \in M$ there is a conformal deformation $\widetilde{g}_{i j}=e^{f} g_{i j}$ of the original metric $g$, such that the new metric is flat, namely, the Riemann tensor associated to $\widetilde{g}$ is zero in $U_{p}$ (here $f: U_{p} \rightarrow \mathbb{R}$ is a smooth function defined in a open neighborhood $U_{p}$ of $p$ ).

\section{SHORT TIME EXISTENCE}

Theorem 2.1. Let $\rho<1 / 2(n-1)$. Then, the evolution equation (1.1) has a unique solution for a positive time interval on any smooth, $n$-dimensional, compact Riemannian manifold $M$ (without boundary) with any initial metric $g_{0}$. 
Proof. We first compute the linearized operator $D L_{g_{0}}$ of the operator $L=-2(\mathrm{Ric}-\rho \mathrm{R} g)$ at a metric $g_{0}$. The Ricci tensor and the scalar curvature have the following linearizations, see [2, Theorem 1.174] or [28], where we use the metric $g_{0}$ to lower and raise indices and to take traces,

$$
\begin{aligned}
D \operatorname{Ric}_{g_{0}}(h)_{i k} & =\frac{1}{2}\left(-\Delta h_{i k}-\nabla_{i} \nabla_{k} \operatorname{tr}(h)+\nabla_{i} \nabla^{t} h_{t k}+\nabla_{k} \nabla^{t} h_{i t}\right)+\text { LOT, } \\
D R_{g_{0}}(h) & =-\Delta(\operatorname{tr} h)+\nabla^{s} \nabla^{t} h_{s t}+\mathrm{LOT},
\end{aligned}
$$

here LOT stands for lower order terms.

Then, the linearization of $L$ at $g_{0}$ is given by

$$
\begin{aligned}
D L_{g_{0}}(h)_{i k} & =-2\left(D \operatorname{Ric}_{g_{0}}(h)_{i k}-\rho D \mathrm{R}_{g_{0}}(h)\left(g_{0}\right)_{i k}\right)+2 \rho \mathrm{R}_{g_{0}} h_{i k} \\
& =\Delta h_{i k}+\nabla_{i} \nabla_{k} \operatorname{tr}(h)-\nabla_{i} \nabla^{t} h_{t k}-\nabla_{k} \nabla^{t} h_{i t}-2 \rho\left(\Delta(\operatorname{tr} h)-\nabla^{s} \nabla^{t} h_{s t}\right)+\text { LOT, }
\end{aligned}
$$

for every bilinear form $h \in \Gamma\left(S^{2} M\right)$. Now, we obtain the principal symbol of the linearized operator in the direction of an arbitrary cotangent vector $\xi$ by replacing each covariant derivative $\nabla_{\alpha}$, appearing in the higher order terms, with the corresponding component $\xi_{\alpha}$,

$$
\begin{aligned}
\sigma_{\xi}\left(D L_{g_{0}}\right)(h)_{i k}= & \xi^{t} \xi_{t} h_{i k}+\xi_{i} \xi_{k} \operatorname{tr}_{g_{0}}(h)-\xi_{i} \xi^{t} h_{k t}-\xi_{k} \xi^{t} h_{i t} \\
& -2 \rho \xi^{t} \xi_{t} \operatorname{tr}_{g_{0}}(h)\left(g_{0}\right)_{i k}+2 \rho \xi^{t} \xi^{s} h_{t s}\left(g_{0}\right)_{i k} .
\end{aligned}
$$

As usual, since the symbol is homogeneous we can assume that $|\xi|_{g_{0}}=1$ and we perform all the computations in an orthonormal basis $\left\{e_{i}\right\}_{i=1, \ldots, n}$ of $T_{p} M$ such that $\xi=$ $g_{0}\left(e_{1}, \cdot\right)$, that is $\xi_{i}=0$ for $i \neq 1$.

Hence, we obtain,

$$
\sigma_{\xi}\left(D L_{g_{0}}\right)(h)_{i k}=h_{i k}+\delta_{i 1} \delta_{k 1} \operatorname{tr}_{g_{0}}(h)-\delta_{i 1} h_{k 1}-\delta_{k 1} h_{i 1}-2 \rho \operatorname{tr}_{g_{0}}(h) \delta_{i k}+2 \rho h_{11} \delta_{i k} .
$$

that can be represented, in the coordinates system

$$
\left(h_{11}, h_{22}, \ldots, h_{n n}, h_{12}, \ldots, h_{1 n}, h_{23}, h_{24}, \ldots, h_{n-1, n}\right)
$$

for any $h \in \Gamma\left(S^{2} M\right)$, by the following matrix

$$
\sigma_{\xi}\left(D L_{g_{0}}\right)=\left(\begin{array}{ccc|c|c}
0 & 1-2 \rho & \ldots & 1-2 \rho & 0 \\
\vdots & A[n-1] & & 0 & 0 \\
0 & 0 & 0 & 0 \\
\hline & 0 & 0 & \operatorname{Id}_{(n-1)(n-2) / 2}
\end{array}\right)
$$


where $A[n-1]$ is the $(n-1) \times(n-1)$ matrix given by

$$
A[n-1]=\left(\begin{array}{cccc}
1-2 \rho & -2 \rho & \ldots & -2 \rho \\
-2 \rho & 1-2 \rho & \ldots & -2 \rho \\
\vdots & \vdots & \ddots & \vdots \\
-2 \rho & -2 \rho & \ldots & 1-2 \rho
\end{array}\right) .
$$

We can see that there are at least $n$ null eigenvalues, as it should be expected by the diffeomorphisms invariance of the operator $L$, and $(n-1)(n-2) / 2$ eigenvalues equal to 1 . The remaining $n-1$ eigenvalues can be computed by the following lemma which is easily proved by induction on the dimension of $A$.

Lemma 2.2. Let $A[m]$ be the $m \times m$ matrix

$$
A[m]=\left(\begin{array}{cccc}
1-2 \rho & -2 \rho & \ldots & -2 \rho \\
-2 \rho & 1-2 \rho & \ldots & -2 \rho \\
\vdots & \vdots & \ddots & \vdots \\
-2 \rho & -2 \rho & \ldots & 1-2 \rho
\end{array}\right) .
$$

Then, there holds

$$
\operatorname{det}\left(A[m]-\lambda \operatorname{Id}_{m}\right)=(1-\lambda)^{(m-1)}(1-2 m \rho-\lambda) .
$$

Using this lemma, we conclude that the eigenvalues of the principal symbol of $D L_{g_{0}}$ are 0 with multiplicity $n, 1$ with multiplicity $\frac{(n+1)(n-2)}{2}$ and $1-2(n-1) \rho$ with multiplicity 1.

Now we apply the so-called DeTurck's trick $[13,14]$ to show that the RB flow is equivalent to a Cauchy problem for a strictly parabolic operator, modulo the action of the diffeomorphism group of $M$. Let $V: \Gamma\left(S^{2} M\right) \rightarrow \Gamma(T M)$ be "DeTurck's" vector field defined by

$$
V^{j}(g)=-g_{0}^{j k} g^{p q} \nabla_{p}\left(\frac{1}{2} \operatorname{tr}_{g}\left(g_{0}\right) g_{q k}-\left(g_{0}\right)_{q k}\right)=-\frac{1}{2} g_{0}^{j k} g^{p q}\left(\nabla_{k}\left(g_{0}\right)_{p q}-\nabla_{p}\left(g_{0}\right)_{q k}-\nabla_{q}\left(g_{0}\right)_{p k}\right),
$$

where $g_{0}$ is a fixed Riemannian metric on $M$ and $g_{0}^{j k}$ are the components of the inverse matrix of $g_{0}$.

The DeTurck's trick (see $[13,14]$ for details) states that in order to show the smooth existence part of the theorem, we only need to check that the operator $D\left(L-\mathscr{L}_{V}\right)_{g_{0}}$ is strongly elliptic, where $\mathscr{L}_{V}$ is the Lie derivative operator in the direction of $V$.

The principal symbol of this latter operator, with the same notations used above, is well known and is given by

$$
\sigma_{\xi}\left(D \mathscr{L}_{V}\right)_{g_{0}}(h)_{i k}=\delta_{i 1} \delta_{k 1} \operatorname{tr}_{g_{0}}(h)-\delta_{i 1} h_{k 1}-\delta_{k 1} h_{i 1} .
$$

Then, we can easily see that the linearized DeTurck-Ricci-Bourguignon operator has principal symbol in the direction $\xi$, with respect to an orthonormal basis $\left\{\xi^{b}, e_{2}, \ldots, e_{n}\right\}$, 
given by

$$
\sigma_{\xi}\left(\left(D\left(L-\mathscr{L}_{V}\right)_{g_{0}}\right)=\left(\begin{array}{cc|c|c}
1 & -2 \rho & \ldots & -2 \rho \\
\vdots & A[n-1] & 0 & 0 \\
0 & 0 & \operatorname{Id}_{(n-1)} & 0 \\
\hline 0 & 0 & \operatorname{Id}_{(n-1)(n-2) / 2}
\end{array}\right),\right.
$$

expressed in the coordinates system

$$
\left(h_{11}, h_{22}, \ldots, h_{n n}, h_{12}, h_{13}, \ldots, h_{1 n}, h_{23}, h_{24}, \ldots, h_{n-1, n}\right)
$$

for any $h \in \Gamma\left(S^{2} M\right)$.

Using Lemma 2.2 again, this matrix has $\frac{n(n+1)}{2}-1$ eigenvalues equals to 1 and 1 eigenvalue equal to $1-2(n-1) \rho$. Therefore, by the DeTurck's trick, a sufficient condition for the existence of a solution is that $\rho<\frac{1}{2(n-1)}$.

The uniqueness part of the theorem is proven in the same way as for the Ricci flow (see [20]). The RB flow is equivalent, via the one parameter group of diffeomorphisms generated by DeTurck's vector field, to the DeTurck-RB flow which is strictly parabolic. On the other hand, the one parameter group of diffeomorphisms satisfies the harmonic map flow introduced by Eells and Sampson in [15], which is also parabolic. These two facts allow to state the uniqueness of the solution for the RB flow (see [11, Chapter 3, Section 4] for more details).

\section{Evolution OF THE CURVATURE}

As the metric tensor evolves as

$$
\frac{\partial}{\partial t} g_{i j}=-2\left(\mathrm{R}_{i j}-\rho \mathrm{R} g_{i j}\right)
$$

it is easy to see, differentiating the identity $g_{i j} g^{j l}=\delta_{i}^{l}$, that

$$
\frac{\partial}{\partial t} g^{j l}=2\left(\operatorname{Ric}^{j l}-\rho \mathrm{R} g^{j l}\right)
$$

It follows that the canonical volume measure $\mu$ satisfies

$$
\frac{d \mu}{d t}=\frac{\partial}{\partial t} \sqrt{\operatorname{det} g_{i j}} \mathscr{L}^{n}=\frac{\sqrt{\operatorname{det} g_{i j}} g^{i j} \frac{\partial}{\partial t} g_{i j}}{2} \mathscr{L}^{n}=(n \rho-1) \mathrm{R} \sqrt{\operatorname{det} g_{i j}} \mathscr{L}^{n}=(n \rho-1) \mathrm{R} \mu .
$$


Computing in a normal coordinates system, the evolution equation for the Christoffel symbols is given by

$$
\begin{aligned}
\frac{\partial}{\partial t} \Gamma_{j k}^{i}= & \frac{1}{2} g^{i l}\left\{\frac{\partial}{\partial x_{j}}\left(\frac{\partial}{\partial t} g_{k l}\right)+\frac{\partial}{\partial x_{k}}\left(\frac{\partial}{\partial t} g_{j l}\right)-\frac{\partial}{\partial x_{l}}\left(\frac{\partial}{\partial t} g_{j k}\right)\right\} \\
& +\frac{1}{2} \frac{\partial}{\partial t} g^{i l}\left\{\frac{\partial}{\partial x_{j}} g_{k l}+\frac{\partial}{\partial x_{k}} g_{j l}-\frac{\partial}{\partial x_{l}} g_{j k}\right\} \\
= & \frac{1}{2} g^{i l}\left\{\nabla_{j}\left(\frac{\partial}{\partial t} g_{k l}\right)+\nabla_{k}\left(\frac{\partial}{\partial t} g_{j l}\right)-\nabla_{l}\left(\frac{\partial}{\partial t} g_{j k}\right)\right\} \\
= & -g^{i l}\left\{\nabla_{j}\left(\mathrm{R}_{k l}-\rho \mathrm{R} g_{k l}\right)+\nabla_{k}\left(\mathrm{R}_{j l}-\rho \mathrm{R} g_{j l}\right)-\nabla_{l}\left(\mathrm{R}_{j k}-\rho \mathrm{R} g_{j k}\right)\right\} \\
= & -\nabla_{j} \mathrm{R}_{k}^{i}-\nabla_{k} \mathrm{R}_{j}^{i}-\nabla^{i} \mathrm{R}_{j k}+\rho\left(\nabla_{j} \mathrm{R} \delta_{k}^{i}+\nabla_{k} \mathrm{R} \delta_{j}^{i}+\nabla^{i} \mathrm{R} g_{j k}\right) .
\end{aligned}
$$

Proposition 3.1. Along the RB flow on a n-dimensional Riemannian manifold $(M, g)$, the curvature tensor, the Ricci tensor and the scalar curvature satisfy the following evolution equations

$$
\begin{aligned}
\frac{\partial}{\partial t} \mathrm{R}_{i j k l}= & \Delta \mathrm{R}_{i j k l}+2\left(\mathrm{~B}_{i j k l}-\mathrm{B}_{i j l k}-\mathrm{B}_{i l j k}+\mathrm{B}_{i k j l}\right) \\
& -g^{p q}\left(\mathrm{R}_{p j k l} \mathrm{R}_{q i}+\mathrm{R}_{i p k l} \mathrm{R}_{q j}+\mathrm{R}_{i j p l} \mathrm{R}_{q k}+\mathrm{R}_{i j k p} \mathrm{R}_{q l}\right) \\
& -\rho\left(\nabla_{i} \nabla_{k} \mathrm{R} g_{j l}-\nabla_{i} \nabla_{l} \mathrm{R} g_{j k}-\nabla_{j} \nabla_{k} \mathrm{R} g_{i l}+\nabla_{j} \nabla_{l} \mathrm{R} g_{i k}\right) \\
& +2 \rho \mathrm{R}_{i j k l},
\end{aligned}
$$

where the tensor $\mathrm{B}$ is defined as $\mathrm{B}_{i j k l}=g^{p q} g^{r s} \mathrm{R}_{i p j r} \mathrm{R}_{k q l s}$.

$$
\begin{aligned}
\frac{\partial}{\partial t} \mathrm{R}_{i k}= & \Delta \mathrm{R}_{i k}+2 g^{p q} g^{r s} \mathrm{R}_{p i r k} \mathrm{R}_{q s}-2 g^{p q} \mathrm{R}_{p i} \mathrm{R}_{q k} \\
& -(n-2) \rho \nabla_{i} \nabla_{k} \mathrm{R}-\rho \Delta \mathrm{R} g_{i k}, \\
\frac{\partial}{\partial t} \mathrm{R}= & (1-2(n-1) \rho) \Delta \mathrm{R}+2|\mathrm{Ric}|^{2}-2 \rho \mathrm{R}^{2} .
\end{aligned}
$$

Proof. The following computation is analogous to the one for the Ricci flow performed by Hamilton [18].

By the first variation formula for the $(4,0)$-Riemann tensor (see [2, Theorem 1.174] or [28]), we have in general

$$
\begin{aligned}
\frac{\partial}{\partial t} \operatorname{Riem}(X, Y, W, Z) & =\frac{1}{2}(h(\operatorname{Riem}(X, Y) W, Z)-h(\operatorname{Riem}(X, Y) Z, W)) \\
- & \frac{1}{2}\left(-\nabla_{Y, W}^{2} h(X, Z)-\nabla_{X, Z}^{2} h(Y, W)+\nabla_{X, W}^{2} h(Y, Z)+\nabla_{Y, Z}^{2} h(X, W)\right),
\end{aligned}
$$


where $X, Y, W, Z \in \Gamma(T M)$ are vector fields and $h=\frac{\partial}{\partial t} g$.

Along the RB flow $h=-2(\operatorname{Ric}-\rho \mathrm{R} g)$, therefore

$$
\begin{aligned}
\frac{\partial}{\partial t} \operatorname{Riem}(X, Y, W, Z)=-\operatorname{Ric}(\operatorname{Riem}(X, Y) W, Z)+\operatorname{Ric}(\operatorname{Riem}(X, Y) Z, W) \\
-\nabla_{Y, W}^{2} \operatorname{Ric}(X, Z)-\nabla_{X, Z}^{2} \operatorname{Ric}(Y, W)+\nabla_{X, W}^{2} \operatorname{Ric}(Y, Z)+\nabla_{Y, Z}^{2} \operatorname{Ric}(X, W) \\
-\rho\left(-\nabla_{Y, W}^{2} \operatorname{Rg}(X, Z)-\nabla_{X, Z}^{2} \operatorname{Rg}(Y, W)+\nabla_{X, W}^{2} \operatorname{Rg}(Y, Z)+\nabla_{Y, Z}^{2} \operatorname{Rg}(X, W)\right) \\
+2 \rho \operatorname{RRiem}(X, Y, W, Z) .
\end{aligned}
$$

Using the second Bianchi identity and the commutation formula for second covariant derivatives, we obtain the following equation for the Laplacian of the Riemann tensor,

$$
\begin{aligned}
\Delta \operatorname{Riem}(X, Y, W, X)= & -\nabla_{Y, W}^{2} \operatorname{Ric}(X, Z)-\nabla_{X, Z}^{2} \operatorname{Ric}(Y, W) \\
& +\nabla_{X, W}^{2} \operatorname{Ric}(Y, Z)+\nabla_{Y, Z}^{2} \operatorname{Ric}(X, W) \\
& -\operatorname{Ric}(\operatorname{Riem}(W, Z) Y, X)+\operatorname{Ric}(\operatorname{Riem}(W, Z) X, Y) \\
& -2(\mathrm{~B}(X, Y, W, Z)-\mathrm{B}(X, Y, Z, W) \\
& +\mathrm{B}(X, W, Y, Z)-\mathrm{B}(X, Z, Y, W)) .
\end{aligned}
$$

Plugging it in the evolution equation, we obtain

$$
\begin{aligned}
\frac{\partial}{\partial t} \operatorname{Riem}(X, Y, W, Z)= & \Delta \operatorname{Riem}(X, Y, W, Z)-\rho\left(\nabla^{2} \mathrm{R} \otimes g\right)(X, Y, W, Z) \\
& +2(\mathrm{~B}(X, Y, W, Z)-\mathrm{B}(X, Y, Z, W) \\
& +\mathrm{B}(X, W, Y, Z)-\mathrm{B}(X, Z, Y, W)) \\
& -\operatorname{Ric}(\operatorname{Riem}(X, Y) W, Z)+\operatorname{Ric}(\operatorname{Riem}(X, Y) Z, W) \\
& -\operatorname{Ric}(\operatorname{Riem}(W, Z) X, Y)+\operatorname{Ric}(\operatorname{Riem}(W, Z) Y, X) \\
& +2 \rho \operatorname{RRiem}(X, Y, W, Z),
\end{aligned}
$$

which is formula (3.2) once written in coordinates. Here the symbol $\oslash$ denotes the Kulkarni-Nomizu product of two symmetric bilinear forms $p$ and $q$, defined by

$(p \otimes q)(X, Y, Z, T)=p(X, Z) q(Y, T)+p(Y, T) q(X, Z)-p(X, T) q(Y, Z)-p(Y, Z) q(X, T)$, for every tangent vectors fields $X, Y, Z, T \in \Gamma(T M)$.

Taking into account the evolution equation for the inverse of the metric (3.1), contracting equation (3.2) and using again the second Bianchi identity, formula (3.3) follows (see [18] for details). Contracting again one gets the evolution equation (3.4) for the scalar curvature.

\subsection{The Uhlenbeck's trick and the evolution of the curvature operator.}

In this subsection we want to study the evolution equation of the curvature operator, as it was done for the Ricci flow by Hamilton in [19].

First of all, we simplify the expression of the reaction term in equation (3.2) by means of the so called Uhlenbeck's trick [19]. Briefly, we will relate the curvature tensor of the evolving metric to an evolving tensor of an abstract bundle with the same symmetries 
of the curvature (see Proposition 3.4) and a nicer evolution equation; afterwards we will find a suitable orthonormal moving frame of $(T M, g(t))$ and write the evolution equation of the coordinates of the Riemann tensor with respect to this frame. The result will be a system of scalar evolution equations and no more a tensorial equation, (see [11] for more details on this method in the case of Ricci flow).

Let $(M, g(t))_{t \in[0, T)}$ be the solution of the RB flow with initial data $g_{0}$ and consider on the tangent bundle $T M$ the family of endomorphisms $\{\varphi(t)\}_{t \in[0, T)}$ defined by the following evolution equation

$$
\left\{\begin{array}{l}
\frac{\partial}{\partial t} \varphi(t)=\operatorname{Ric}_{g(t)}^{\#} \circ \varphi(t)-\rho \mathrm{R}_{g(t)} \varphi(t), \\
\varphi(0)=\operatorname{Id}_{T M},
\end{array}\right.
$$

where $\operatorname{Ric}_{g(t)}^{\#}$ is the endomorphism of the tangent bundle canonically associated to the Ricci tensor by raising an index.

For every point $p$ of the manifold $M$, the evolution equation (3.5) represents a system of linear ODEs on the fiber $T_{p} M$, therefore a unique solution exists as long as the RB flow exists. Moreover, if we let $(h(t))_{t \in[0, T)}$ be the family of bundle metrics defined by $h(t)=\varphi(t)^{*}(g(t))$, where $\varphi(t)$ satisfies system (3.5), then $h(t)=g_{0}$ for every $t \in[0, T)$. As

$$
\forall t \in[0, T), \quad \varphi(t):\left(T M, g_{0}\right) \rightarrow(T M, g(t))
$$

is a bundle isometry, the pull-back via $\varphi(t)$ of the Levi-Civita connection associated to $g(t)$ is a connection on $T M$ compatible with the metric $g_{0}$. In the following, we will denote by $(V, h)$ the vector bundle $\left(T M, g_{0}\right)$ in order to stress out the fact that we are not considering the Levi-Civita connection associated to $g_{0}$, but the family of timedependent connections $D(t)$ defined via the bundle isometries $\varphi(t)$.

The following lemma states some basic properties of these pull-back connections.

Lemma 3.2. (see [11, Chapter 6, Section 2] Let $D(t): \Gamma(T M) \times \Gamma(V) \rightarrow \Gamma(V)$ be the pullback connection defined by

$$
D(t)_{X} \zeta=\varphi(t)^{*}\left(\nabla_{X}^{g(t)}(\varphi(t)(\zeta))\right)
$$

$\forall t \in[0, T), \forall X \in \Gamma(T M), \forall \zeta \in \Gamma(V)$, where $\nabla^{g(t)}$ is the Levi-Civita connection of $g(t)$.

Let again $D(t)$ be the canonical extension to the tensor powers of $V$ and $T$ be a covariant tensor on $M$. Then, for every $t \in[0, T)$ and $X \in \Gamma(T M)$ there holds

$$
D(t)_{X}\left(\varphi(t)^{*}(T)\right)=\varphi(t)^{*}\left(\nabla_{X}^{g(t)} T\right) .
$$

In particular, $D(t)_{X} h=\varphi^{*}\left(\nabla_{X}^{g(t)} g(t)\right)=0$, so every connection of the family $D(t)$ is compatible with the bundle metric $h$ on $V$.

Let $D^{2}: \Gamma(T M) \times \Gamma(T M) \times \Gamma(V) \rightarrow \Gamma(V)$ be the second covariant derivative defined by

$$
D_{X, Y}^{2}(\zeta)=D_{X}\left(D_{Y} \zeta\right)-D_{\nabla_{X}^{g(t)} Y} \zeta, \quad \forall X, Y \in \Gamma(T M), \forall \zeta \in \Gamma(V),
$$


and the rough Laplacian defined by $\Delta_{D}=\operatorname{tr}_{g}\left(D^{2}\right)$. Then, for every covariant tensor $T$ on $M$, there hold

$$
\begin{aligned}
D_{X, Y}^{2}\left(\varphi^{*}(T)\right) & =\varphi^{*}\left(\nabla_{X, Y}^{2} T\right) \quad \forall X, Y \in \Gamma(T M), \\
\Delta_{D}\left(\varphi^{*}(T)\right) & =\varphi^{*}\left(\Delta_{g} T\right) .
\end{aligned}
$$

Remark 3.3. Let $\mathcal{R} \in \operatorname{End}\left(\Lambda^{2} M\right)$ be the Riemann curvature operator defined by

$$
\langle\mathcal{R}(X \wedge Y), W \wedge Z\rangle=\operatorname{Riem}(X, Y, W, Z),
$$

where $\langle$,$\rangle is the linear extension of g$ to the exterior powers of $T M$.

In the following, we use a convention on the Lie algebra structure of $\Lambda^{2} M$ which is different from the original one chosen by Hamilton in [19]. More precisely, with his convention the eigenvalues of the curvature operator are twice the sectional curvatures, whereas with our convention the curvature operator has the sectional curvatures as eigenvalues. In particular, every formula differs from the corresponding one in the usual theory of the Ricci flow by a factor 2 (see also [11, Chapter 6, Section 3] for the details). We recall that $\mathcal{R}$ can be considered as an element of $\Gamma\left(S^{2}\left(\Lambda^{2} M\right)\right)$, and the following equations hold true

$$
\begin{aligned}
\mathrm{R} & =2 \sum_{i<k} \mathcal{R}_{(i k)}^{(i k)} ; \\
\left(\mathcal{R}^{2}\right)_{i j k l} & =\mathrm{B}_{i j k l}-\mathrm{B}_{i j l k} ; \\
(\mathcal{R} \# \mathcal{R})_{i j k l} & =\mathrm{B}_{i k j l}-\mathrm{B}_{i l j k} .
\end{aligned}
$$

where B is defined as in Proposition 3.1. For more details on the structure of the curvature operator we refer again the reader to [11, Chapter 6, Section 3].

We now consider the pull-back of the Riemann curvature tensor and the curvature operator.

Proposition 3.4. Let Piem be the pull-back of the Riemann curvature tensor via the family of bundle isometries $\{\varphi(t)\}_{t \in[0, T)}$. The following statements hold true:

(1) Piem has the same symmetry properties as Riem, i.e. it can be seen as an element of $\Gamma\left(S^{2}\left(\Lambda^{2} V\right)\right)$ and it satisfies the first Bianchi identity;

(2) For every $p \in M$ and $t \in[0, T)$ the algebraic curvature operator $\mathcal{P}(p, t) \in \operatorname{End}\left(\Lambda^{2} V_{p}\right)$ (see Remark 3.7), defined by $\varphi \circ \mathcal{P}=\mathcal{R} \circ \varphi$ has the same eigenvalues as $\mathcal{R}(p, t)$. In particular, $\mathcal{P}$ is positive (nonnegative) definite if and only if $\mathcal{R}$ is positive (nonnegative) definite;

(3) $\operatorname{Pic}(t)=\operatorname{tr}_{h}(\operatorname{Piem}(t))=\varphi(t)^{*}\left(\operatorname{Ric}_{g(t)}\right)$;

(4) $\mathrm{P}=\operatorname{tr}_{h}(\operatorname{Pic}(t))=\mathrm{R}_{g(t)}$;

(5) $\mathrm{B}(\mathrm{Piem})=\varphi^{*}(\mathrm{~B}($ Riem $))$, where $\mathrm{B}$ is defined in the same way as in Proposition 3.1 for a generic element of $S^{2}\left(\Lambda^{2} V\right)$.

Finally, we can compute the evolution of Piem and $\mathcal{P}$. 
Proposition 3.5. The tensors Piem and $\mathcal{P}$ satisfy respectively the following evolution equations

$$
\begin{aligned}
\frac{\partial}{\partial t}(\text { Piem })_{a b c d}= & \Delta_{D}(\text { Piem })_{a b c d}-\rho\left(\varphi^{*}\left(\nabla^{2} \mathrm{R}\right) \otimes h\right)_{a b c d} \\
& +2\left(\mathrm{~B}(\text { Piem })_{a b c d}-\mathrm{B}(\text { Piem })_{a b d c}+\mathrm{B}(\text { Piem })_{a c b d}-\mathrm{B}(\text { Piem })_{a d b c}\right) \\
& -2 \rho \mathrm{P} \mathrm{Piem} \operatorname{Pabcd}_{a}, \\
\frac{\partial}{\partial t} \mathcal{P}= & \Delta_{D} \mathcal{P}-2 \rho \varphi^{*}\left(\nabla^{2} \operatorname{tr}_{h}(\mathcal{P})\right) \otimes h+2 \mathcal{P}^{2}+2 \mathcal{P}^{\#}-4 \rho \operatorname{tr}_{h}(\mathcal{P}) \mathcal{P},
\end{aligned}
$$

where $\operatorname{tr}_{h}(\mathcal{P}(t))=\operatorname{tr}_{g(t)}(\mathcal{R}(t))=\frac{1}{2} \mathrm{R}(t)$.

Remark 3.6. On the right hand side of equation (3.9) the term $\varphi^{*}\left(\nabla^{2} \mathrm{R}\right)$ appears (i.e. the pull-back of the Hessian of the scalar curvature, seen as a symmetric 2 -form on the tangent bundle) and it cannot be expressed in terms of the connection $D(t)$.

Proof. Let $\zeta_{1}, \ldots, \zeta_{4}$ be sections of $V$; then combining the evolution equations of the Riemann tensor (3.2) and of the bundle isometry $\varphi$ (3.5), we obtain

$$
\begin{aligned}
& \frac{\partial}{\partial t}(\operatorname{Piem})\left(\zeta_{1}, \zeta_{2}, \zeta_{3}, \zeta_{4}\right)= \\
& \varphi^{*}\left(\frac{\partial}{\partial t} \operatorname{Riem}\right)\left(\zeta_{1}, \zeta_{2}, \zeta_{3}, \zeta_{4}\right)+\operatorname{Riem}\left(\frac{\partial \varphi}{\partial t}\left(\zeta_{1}\right), \varphi\left(\zeta_{2}\right), \varphi\left(\zeta_{3}\right), \varphi\left(\zeta_{4}\right)\right) \\
& +\operatorname{Riem}\left(\varphi\left(\zeta_{1}\right), \frac{\partial \varphi}{\partial t}\left(\zeta_{2}\right), \varphi\left(\zeta_{3}\right), \varphi\left(\zeta_{4}\right)\right)+\operatorname{Riem}\left(\varphi\left(\zeta_{1}\right), \varphi\left(\zeta_{2}\right), \frac{\partial \varphi}{\partial t}\left(\zeta_{3}\right), \varphi\left(\zeta_{4}\right)\right) \\
& +\operatorname{Riem}\left(\varphi\left(\zeta_{1}\right), \varphi\left(\zeta_{2}\right), \varphi\left(\zeta_{3}\right), \frac{\partial \varphi}{\partial t}\left(\zeta_{4}\right)\right) \\
& =\varphi^{*}\left(\Delta_{g} \operatorname{Riem}\right)\left(\zeta_{1}, \zeta_{2}, \zeta_{3}, \zeta_{4}\right)-\rho \varphi^{*}\left(\nabla^{2} \mathrm{R} \otimes g\right)\left(\zeta_{1}, \zeta_{2}, \zeta_{3}, \zeta_{4}\right) \\
& +2 \varphi^{*}\left(\mathrm{~B}(\operatorname{Riem})\left(\zeta_{1}, \zeta_{2}, \zeta_{3}, \zeta_{4}\right)-\mathrm{B}(\operatorname{Riem})\left(\zeta_{1}, \zeta_{2}, \zeta_{4}, \zeta_{3}\right)-\mathrm{B}(\operatorname{Riem})\left(\zeta_{1}, \zeta_{4}, \zeta_{2}, \zeta_{3}\right)\right. \\
& \left.+\operatorname{B}(\operatorname{Riem})\left(\zeta_{1}, \zeta_{3}, \zeta_{2}, \zeta_{4}\right)\right)+2 \rho \operatorname{R} \varphi^{*}(\operatorname{Riem})\left(\zeta_{1}, \zeta_{2}, \zeta_{3}, \zeta_{4}\right) \\
& -\operatorname{Riem}\left(\operatorname{Ric}^{\#} \circ \varphi\left(\zeta_{1}\right), \varphi\left(\zeta_{2}\right), \varphi\left(\zeta_{3}\right), \varphi\left(\zeta_{4}\right)\right)-\operatorname{Riem}\left(\varphi\left(\zeta_{1}\right), \operatorname{Ric}^{\#} \circ \varphi\left(\zeta_{2}\right), \varphi\left(\zeta_{3}\right), \varphi\left(\zeta_{4}\right)\right) \\
& -\operatorname{Riem}\left(\varphi\left(\zeta_{1}\right), \varphi\left(\zeta_{2}\right), \operatorname{Ric}^{\#} \circ \varphi\left(\zeta_{3}\right), \varphi\left(\zeta_{4}\right)\right)-\operatorname{Riem}\left(\varphi\left(\zeta_{1}\right), \varphi\left(\zeta_{2}\right), \varphi\left(\zeta_{3}\right), \operatorname{Ric}^{\#} \circ \varphi\left(\zeta_{4}\right)\right) \\
& +\operatorname{Riem}\left(\left(\operatorname{Ric}^{\#} \circ \varphi-\rho \operatorname{R} \varphi\right)\left(\zeta_{1}\right), \varphi\left(\zeta_{2}\right), \varphi\left(\zeta_{3}\right), \varphi\left(\zeta_{4}\right)\right) \\
& +\operatorname{Riem}\left(\varphi\left(\zeta_{1}\right),\left(\operatorname{Ric}^{\#} \circ \varphi-\rho \operatorname{R} \varphi\right)\left(\zeta_{2}\right), \varphi\left(\zeta_{3}\right), \varphi\left(\zeta_{4}\right)\right) \\
& +\operatorname{Riem}\left(\varphi\left(\zeta_{1}\right), \varphi\left(\zeta_{2}\right),\left(\operatorname{Ric}^{\#} \circ \varphi-\rho \operatorname{R} \varphi\right)\left(\zeta_{3}\right), \varphi\left(\zeta_{4}\right)\right) \\
& +\operatorname{Riem}\left(\varphi\left(\zeta_{1}\right), \varphi\left(\zeta_{2}\right), \varphi\left(\zeta_{3}\right),\left(\operatorname{Ric}^{\#} \circ \varphi-\rho \operatorname{R} \varphi\right)\left(\zeta_{4}\right)\right) \\
& =\Delta_{D}(\mathrm{Piem})\left(\zeta_{1}, \zeta_{2}, \zeta_{3}, \zeta_{4}\right)-\rho\left(\varphi^{*}\left(\nabla^{2} \mathrm{R}\right) \otimes h\right)\left(\zeta_{1}, \zeta_{2}, \zeta_{3}, \zeta_{4}\right) \\
& +2 \varphi^{*}\left(\mathrm{~B}(\mathrm{Piem})\left(\zeta_{1}, \zeta_{2}, \zeta_{3}, \zeta_{4}\right)-\mathrm{B}(\mathrm{Piem})\left(\zeta_{1}, \zeta_{2}, \zeta_{4}, \zeta_{3}\right)-\mathrm{B}(\mathrm{Piem})\left(\zeta_{1}, \zeta_{4}, \zeta_{2}, \zeta_{3}\right)\right. \\
& \left.+\mathrm{B}(\operatorname{Piem})\left(\zeta_{1}, \zeta_{3}, \zeta_{2}, \zeta_{4}\right)\right)-2 \rho \operatorname{PPiem}\left(\zeta_{1}, \zeta_{2}, \zeta_{3}, \zeta_{4}\right),
\end{aligned}
$$


where we used several identities stated above. For $\zeta_{1}, \ldots, \zeta_{4}$ belonging to a local frame we get the desired equation (3.9).

Combining the evolution equation for Piem with the formulas stated in Remark 3.3, we find the evolution equation of $\mathcal{P}$.

Remark 3.7. It must be noticed that, even though for every $p \in M$ and $t \in[0, T), \mathcal{P}(p, t)$ belongs to the set of algebraic curvature operators $\mathcal{C}_{b}\left(V_{p}\right)$, in general it does not coincide with the curvature operator of the pull-back connection $D(t)$. In the present literature the pull-back tensor is always denoted by Riem and this abuse of notation is somehow misleading, suggesting the wrong impression that $\operatorname{Piem}(t)=\varphi(t)^{*}\left(\operatorname{Riem}_{g(t)}\right)$ is equal to $\operatorname{Riem}_{\varphi(t)^{*}(g(t))}=\operatorname{Riem}_{h}$, but this is not longer true for general isomorphisms of the tangent bundle (however it is true for $\varphi \in \operatorname{Dif} f(M)$ ).

By the Uhlenbeck's trick the evolution equation (3.10) for $\mathcal{P}$ allows a simpler use of the maximum principle for tensor as the reaction term is nicer and the metric on $S^{2}\left(\Lambda^{2} V\right)$ is independent of time. Moreover, the subsets of $S^{2}\left(\Lambda^{2} V\right)$ preserved by such PDE correspond to curvature conditions preserved under the RB flow.

\section{PRESERVED CURVATURE CONDITIONS}

In this section we will use the maximum principle in various formulations in order to find curvature conditions which are preserved by the RB flow.

We begin by considering the evolution equation for the scalar curvature (3.4), which behaves as under the Ricci flow.

Proposition 4.1. Let $(M, g(t))_{t \in[0, T)}$ be a compact maximal solution of the RB flow (1.1). If $\rho \leq \frac{1}{2(n-1)}$, the minimum of the scalar curvature is nondecreasing along the flow. In particular if $\mathrm{R}(g(0)) \geq \alpha$, for some $\alpha \in \mathbb{R}$, then $\mathrm{R}_{g(t)} \geq \alpha$ for every $t \in[0, T)$. Moreover if $\alpha>0$ then $T \leq \frac{n}{2(1-n \rho) \alpha}$.

Proof. As $\rho \leq \frac{1}{2(n-1)} \leq \frac{1}{n}$, for any $n>1$, it follows that

$$
\begin{aligned}
\frac{\partial}{\partial t} \mathrm{R} & =(1-2(n-1) \rho) \Delta \mathrm{R}+2|\mathrm{Ric}|^{2}-2 \rho \mathrm{R}^{2} \\
& \geq(1-2(n-1) \rho) \Delta \mathrm{R}+2 \mathrm{R}^{2} / n-2 \rho \mathrm{R}^{2} \\
& \geq(1-2(n-1) \rho) \Delta \mathrm{R},
\end{aligned}
$$

hence, by the maximum principle, the minimum of the scalar curvature is nondecreasing along the RB flow on a compact manifold. In particular, for every $\alpha \in \mathbb{R}$, the condition $\mathrm{R} \geq \alpha$ is preserved.

Finally, integrating the inequality

$$
\frac{\partial}{\partial t} \mathrm{R}_{\min } \geq 2\left(\frac{1}{n}-\rho\right) \mathrm{R}_{\min }^{2}
$$


that holds almost everywhere for $t \in[0, T)$ (by the Hamilton's trick (see [21], [24, Lemma 2.1.3])), we obtain

$$
\mathrm{R}_{\text {min }}(t) \geq \frac{n \alpha}{n-2(1-n \rho) \alpha t},
$$

that, for $\alpha>0$, gives the estimate on the maximal time of existence.

Remark 4.2. In the special case of the Schouten flow (when $\rho=\frac{1}{2(n-1)}$ ), actually there holds

$$
\frac{\partial}{\partial t} \mathrm{R} \geq \frac{n-2}{n(n-1)} \mathrm{R}^{2},
$$

at every point of the manifold, which implies that the scalar curvature is pointwise nondecreasing and diverges in finite time.

Remark 4.3. By means of the strong maximum principle, it follows that if the initial manifold has nonnegative scalar curvature then either the manifold is Einstein $(\mathrm{Ric}=0)$ or the scalar curvature becomes positive for every positive time under any RB flow with $\rho \leq \frac{1}{2(n-1)}$.

Proposition 4.4. Let $(M, g(t))_{t \in(-\infty, 0]}$ be a compact, $n$-dimensional, ancient solution of the $R B$ flow (1.1). If $\rho \leq \frac{1}{2(n-1)}$ then, either $\mathrm{R}>0$ or Ric $\equiv 0$ on $M \times(-\infty, 0]$.

Proof. As $g(t)$ is an ancient solution, for every $t_{0}<t_{1} \leq 0$, we can define $\widetilde{g}(s)=g\left(s+t_{0}\right)$, which is a solution of the RB flow for $s \in\left[0, t_{1}-t_{0}\right]$. Then, we have $\widetilde{\mathrm{R}}_{\text {min }}(0)=\mathrm{R}_{\text {min }}\left(t_{0}\right)$, hence, from formula (4.1)

$$
\widetilde{\mathrm{R}}_{\text {min }}(s) \geq \frac{n}{n \widetilde{\mathrm{R}}_{\text {min }}^{-1}(0)-2(1-n \rho) s},
$$

for every $s \in\left(0, t_{1}-t_{0}\right]$. In particular, we have

$$
\mathrm{R}_{\text {min }}\left(t_{1}\right)=\widetilde{\mathrm{R}}_{\text {min }}\left(t_{1}-t_{0}\right) \geq \frac{n}{n \mathrm{R}_{\text {min }}^{-1}\left(t_{0}\right)-2(1-n \rho)\left(t_{1}-t_{0}\right)} .
$$

If $\mathrm{R}_{\text {min }}\left(t_{0}\right) \geq 0$, by Proposition 4.1 , it follows that $\mathrm{R}_{\text {min }}\left(t_{1}\right) \geq 0$, so we can assume that $\mathrm{R}_{\text {min }}\left(t_{0}\right)<0$, hence

$$
\mathrm{R}_{\text {min }}\left(t_{1}\right) \geq \frac{n}{n \mathrm{R}_{\text {min }}^{-1}\left(t_{0}\right)-2(1-n \rho)\left(t_{1}-t_{0}\right)}>-\frac{n}{2(1-n \rho)\left(t_{1}-t_{0}\right)},
$$

for every $t_{1}<t_{0}$, and sending $t_{0}$ to $-\infty$, we still conclude that $\mathrm{R}_{\min }\left(t_{1}\right) \geq 0$. Since this holds for every $t_{1} \leq 0$ the previous remark implies the result.

\subsection{Maximum principle for uniformly elliptic operators.}

Let $M$ be a smooth compact manifold, $g(t), t \in[0, T)$, a family of Riemannian metrics on $M$ and $(E, h(t)) t \in[0, T)$, be a real vector bundle on $M$, endowed with a (possibly time-dependent) bundle metric. Let $D(t): \Gamma(T M) \times \Gamma(E) \rightarrow \Gamma(E)$ be a family of linear connections on $E$ compatible at each time with the bundle metric $h(t)$. We have already seen in Section 3.1 how to define the second covariant derivative, using also the LeviCivita connections $\nabla_{g(t)}$ associated to the Riemannian metrics on $M$. 
Definition 4.5. A second-order linear operator $\mathcal{L}$ on $\Gamma(E)$ can be written in a local frame field $\left\{e_{i}\right\}_{i=1, \ldots, n}$ of $T M$

$$
\mathcal{L}=a^{i j} D_{e_{i} e_{j}}^{2}-b^{i} D_{e_{i}}
$$

where $a=a^{i j} e_{i} \otimes e_{j} \in \Gamma\left(S^{2}(T M)\right)$ is a symmetric $(0,2)$-tensor and $b=b^{i} e_{i}$ is a smooth vector field. We say that $\mathcal{L}$ is uniformly elliptic if $a$ is uniformly positive definite.

Remark 4.6. In the previous definition, both the coefficients and the connections are in general time-dependent and we say that $\mathcal{L}$ is uniformly elliptic if it is so for every $t \in$ $[0, T)$ uniformly in time.

Weinberger in [29] proved the maximum principle for systems of solutions of a timedependent heat equation in the Euclidean space; Hamilton in [19] treated the general case of a vector bundle over an evolving Riemannian manifold. Here we present a slight generalization of Hamilton's theorem for parabolic equations with uniformly elliptic operator (see [26, Theorem 2.2] for the "static" version proved by Savas-Halilaj and Smoczyk).

As before, $(M, g(t))$ is a smooth compact manifold equipped with a family of Riemannian metrics; we consider a real vector bundle $E$ over $M$, equipped with a fixed bundle metric $h$ and a family of time-dependent connections $D(t)$ compatible at every time with $h$.

Definition 4.7. Let $S \subset E$ be a sub-bundle and denote $S_{p}=S \cap E_{p}$ for every $p \in M$. We say that $S$ is invariant under parallel translation w.r.t. $D$, if for every curve $\gamma:[0, l] \rightarrow M$ and vector $v \in S_{\gamma(0)}$, the unique parallel (w.r.t. $D$ ) section $v(s) \in E_{\gamma(s)}$ along $\gamma(s)$ with $v(0)=v$ is contained in $S$.

Theorem 4.8 (Vectorial Maximum Principle). Let $u:[0, T) \rightarrow \Gamma(E)$ be a smooth solution of the following parabolic equation

$$
\frac{\partial}{\partial t} u=\mathcal{L} u+F(u, t)
$$

where $\mathcal{L}$ is a uniformly elliptic operator as defined in (4.2) and $F: E \times[0, T) \rightarrow E$ is a continuous map, locally Lipschitz in the $E$ factor, which is also fiber-preserving, i.e. $F(v, t) \in$ $E_{p}$ for every $p \in M, v \in E_{p}, t \in[0, T)$.

Let $K \subset E$ be a closed sub-bundle (for the metric $h$ ), invariant under parallel translation w.r.t. $D(t)$, for every $t \in[0, T)$, and convex in the fibers, i.e. $K_{p}=K \cap E_{p}$ is convex for every $p \in M$. Suppose that $K$ is preserved by the ODE associated to (4.3), i.e. for every $p \in M$ and $U_{0} \in K_{p}$, the solution $U(t)$ of

$$
\begin{cases}\frac{d U}{d t} & =F_{p}(U(t), t) \\ U(0) & =U_{0}\end{cases}
$$

remains in $K_{p}$. Then, if $u$ is contained in $K$ at time 0 , $u$ remains in $K$, i.e. $u(p, t) \in K_{p}$ for every $p \in M, t \in[0, T)$, as long as the solution of the ODE exists. 
Proof. (Sketch) We can follow exactly the detailed proof written in [10, Chapter 10, Section 3], provided that we generalize [10, Lemma 10.34] to the analogue one for uniformly elliptic operator (see again [26, Lemma 2.2]): if $K \subset E$ satisfies all the hypothesis of Theorem 4.8 and $u \in \Gamma(E)$ is a smooth section of $E$, then

$$
u(p) \in K_{p} \quad \forall p \in M \quad \Longrightarrow \quad \mathcal{L}(u)_{p} \in C_{u(p)} K_{p} \quad \forall p \in M,
$$

where $C_{u(p)} K_{p}$ is the tangent cone of the convex set $K_{p}$ at $u(p)$.

There is a further generalization of this maximum principle which allows the subset $K$ to be time-dependent.

Theorem 4.9 (Vectorial Maximum Principle, Time-dependent Set). Let $u:[0, T) \rightarrow \Gamma(E)$ be a smooth solution of the parabolic equation (4.3), with the notations of the previous Theorem. For every $t \in[0, T)$, let $K(t) \subset E$ be a closed sub-bundle (for the metric $h$ ), invariant under parallel translation w.r.t. $D(t)$, convex in the fibers and such that the space-time track

$$
\mathcal{T}=\{(v, t) \in E \times \mathbb{R}: v \in K(t), t \in[0, T)\}
$$

is closed in $E \times[0, T)$. Suppose that, for every $t_{0} \in[0, T), K\left(t_{0}\right)$ is preserved by the ODE associated, i.e. for any $p \in M$, any solution $U(t)$ of the $O D E$ that starts in $K\left(t_{0}\right)_{p}$ remains in $K(t)_{p}$, as long as it exists. Then, if $u(0)$ is contained in $K(0), u(p, t) \in K(t)_{p}$ for ever $p \in M$, $t \in[0, T)$, as long as the solution of the ODE exists.

The proof of this Theorem is basically a refinement of the arguments used in the others maximum principles (see [10, Chapter 10]).

As remarked before, the evolution equation (3.2) of the Riemann tensor has some mixed product of type Riem $*$ Ric which makes difficult to understand the behavior of the reaction term. On the other hand, if we perform the Uhlenbeck's trick, the evolution equation (3.9) becomes a little nicer and can be used to understand how the RB flow affects the geometry.

More precisely, we use the evolution equation (3.10) for the pull-back of the Riemann curvature operator $\mathcal{P} \in \Gamma\left(S^{2}\left(\Lambda^{2} V^{*}\right)\right)$ to prove that the cone of nonnegative curvature operators is preserved by the RB flow.

Proposition 4.10. Let $(M, g(t))_{t \in[0, T)}$ be a compact solution of the RB flow (1.1) with $\rho<\frac{1}{2(n-1)}$ and such that the initial data $g_{0}$ has nonnegative curvature operator. Then $\mathcal{R}_{g(t)} \geq 0$ for every $t \in[0, T)$.

Proof. We recall the evolution equation (3.10) for $\mathcal{P}=\varphi^{*} \mathcal{R}$

$$
\frac{\partial}{\partial t} \mathcal{P}=\Delta_{D} \mathcal{P}-2 \rho \varphi^{*}\left(\nabla^{2} \operatorname{tr}_{h}(\mathcal{P})\right) \otimes h+2 \mathcal{P}^{2}+2 \mathcal{P} \#-4 \rho \operatorname{tr}_{h}(\mathcal{P}) \mathcal{P},
$$

where $\operatorname{tr}_{h}(\mathcal{P}(t))=1 / 2 \mathrm{R}(t)$ is half of the scalar curvature of the metric $g(t)$. By proposition 3.4, it suffices to show that the non negativity of $\mathcal{P}$ is preserved by equation (3.10). We want to apply the vectorial maximum principle 4.8 , therefore we must show that

$$
\mathcal{L}(Q)=\Delta_{D} Q-2 \rho \varphi^{*}\left(\nabla^{2} \operatorname{tr}_{h}(Q)\right) \oplus h
$$


is a uniformly elliptic operator on the bundle $\left(\Gamma\left(S^{2}\left(\Lambda^{2} V^{*}\right)\right), h, D(t)\right)$.

As $\mathcal{L}$ is a linear second order operator, we compute as usual its principal symbol in the arbitrary direction $\xi$. In order to simplify the computations, we choose opportune frames at every point $p \in M$ and time $t \in[0, T)$. Then, let $\left\{e_{i}\right\}_{i=1, \ldots, n}$ be an orthonormal basis of $\left(V_{p}, h_{p}\right)$ such that $\xi=h_{p}\left(e_{1}, \cdot\right)$. According to the Uhlenbeck's trick (Section 3.1) and the convention on algebraic curvature operators (Section 3.1) we have that $\left\{f_{i}=\right.$ $\left.\varphi(t)_{p}\left(e_{i}\right)\right\}_{i_{1}, \ldots, n}$ is an orthonormal basis of $T_{p} M$ with respect to $g(t)_{p}$, the components of $\varphi(t)_{p}$ with these choices are $\varphi_{i}^{a}=\delta_{i}^{a}$ and $\left\{e_{i} \wedge e_{j}\right\}_{i<j}$ is an orthonormal basis of $\Lambda^{2} V_{p}$. Hence, the principal symbol of the operator $\mathcal{L}$ written in these frames is

$$
\begin{aligned}
\sigma_{\xi}(\mathcal{L} Q)_{(i j)(k l)} & =\xi^{p} \xi_{p} Q_{(i j)(k l)}-2 \rho \delta_{i}^{a} \delta_{j}^{b} \delta_{k}^{c} \delta_{l}^{d} \operatorname{tr}_{h}(Q)(\xi \otimes \xi \otimes h)_{(a b)(c d)} \\
& =|\xi|^{2} Q_{(i j)(k l)}-2 \rho \operatorname{tr}_{h}(Q)(\xi \otimes \xi \otimes h)_{(i j)(k l)} \\
& =Q_{(i j)(k l)}-2 \rho\left(\sum_{p<q} Q_{(p q)(p q)}\right) \delta_{i}^{1} \delta_{k}^{1} \delta_{j l}
\end{aligned}
$$

where we used that $|\xi|=1, i<j$ and $k<l$ in the last passage. Now it is easy to see that the matrix representing the symbol has the following form

$$
\sigma_{\xi}(\mathcal{L})=\left(\begin{array}{c|ccc|c}
-2 \rho & \ldots & 2 \rho & 0 \\
\vdots & \ddots & \vdots & 0 \\
-2 \rho-1] & \ldots & -2 \rho & 0 \\
\hline 0 & \operatorname{Id}_{(n-1)}(n-2) / 2 & \operatorname{Id}_{N(N-1) / 2}
\end{array}\right),
$$

where we have ordered the components as follows: first the $n-1$ ones of the form $(1 j)(1 j)$ with $j>1$, then the $(n-1)(n-2) / 2$ ones of the form $(i j)(i j)$ with $1<i<j$, and last the $N(N-1) / 2$ "non diagonal" ones, with $N=n(n-1) / 2$ and $A$ is the matrix defined in (2.1).

By lemma 2.2 the eigenvalues of the symbol are 1 with multiplicity $N(N+1) / 2-1$ and $1-2(n-1) \rho$ with multiplicity 1 , since $\rho<1 / 2(n-1)$ the operator $\mathcal{L}$ is uniformly elliptic.

In the second part of the proof we consider the reaction term $F(Q)=2\left(Q^{2}+Q^{\#}-\right.$ $\left.2 \rho \operatorname{tr}_{h}(Q) Q\right)$. Clearly $F$ is continuous, locally Lipschitz and fiber-preserving. Let $\Omega \in$ $\Gamma\left(S^{2}\left(\Lambda^{2} V^{*}\right)\right)$ be the set of nonnegative algebraic curvature operators, where we have identified $S^{2}\left(\Lambda^{2} V^{*}\right) \simeq E n d_{S A}\left(\Lambda^{2} V\right)$ via the metric $h$. We observe that $\Omega=\left\{Q: \quad \lambda_{N}\left(Q_{p}\right) \geq\right.$ $0\}$, where $N=n(n-1) / 2$ and $\lambda_{N}$ is the least eigenvalue of $Q_{p}$. Hence $\Omega$ is clearly closed, by [10, Lemma 10.11] it is invariant under parallel translation with respect to every connection $D(t)$ and it is convex, provided that the function $Q \mapsto \lambda_{N}\left(Q_{p}\right)$ is concave. We 
can rewrite

$$
\lambda_{N}\left(Q_{p}\right)=\inf _{\left\{v \in \Lambda^{2} V_{p}:|v|_{h}=1\right\}} h\left(Q_{p}(v), v\right) ;
$$

so it is easy to conclude, by bilinearity of the metric $h$ and concavity of inf, that the function defining $\Omega$ is actually concave and so its superlevels are convex. In order to finish the proof we have to show that the ODE $d Q / d t=F(Q)$ preserves $\Omega$. Now, by standard facts in convex analysis, we only need to prove that

$$
F_{p}\left(Q_{p}\right) \in T_{Q_{p}} \Omega_{p} \quad \text { for every } p \in M \text { such that } Q_{p} \in \partial \Omega_{p}
$$

where $\partial \Omega_{p}=\left\{Q_{p} \in \Omega_{p}: \exists v \in \Lambda^{2} V_{p} \quad Q_{p}(v, v)=0\right\}$ and the tangent cone is

$$
T_{Q_{p}} \Omega_{p}=\left\{S_{p} \in S^{2}\left(\Lambda^{2} V_{p}^{*}\right): S_{p}(v, v) \geq 0 \text { for every } v \in \Lambda^{2} V_{p} \text { such that } Q_{p}(v, v)=0\right\}
$$

Let $v \in \Lambda^{2} V_{p}$ and $\left\{\theta_{\alpha}\right\}$ be respectively a null eigenvector of $Q_{p}$ and an orthonormal basis of $\Lambda^{2} V_{p}$ that diagonalizes $Q_{p}$. Clearly

$$
v=v^{\alpha} \theta_{\alpha}, \quad\left(Q_{p}\right)_{\alpha \beta}=\lambda_{\alpha} \delta_{\alpha \beta} .
$$

with $\lambda_{\alpha} \geq 0$. Then

$$
\left(Q_{p}^{2}\right)_{\alpha \beta}=\lambda_{\alpha}^{2} \delta_{\alpha \beta}, \quad\left(Q_{p}^{\#}\right)_{\alpha \beta}=\frac{1}{2}\left(c_{\alpha}^{\gamma \nu}\right)^{2} \lambda_{\gamma} \lambda_{\nu} \delta_{\alpha \beta}
$$

and

$$
F_{p}\left(Q_{p}\right)(v, v)=\lambda_{\alpha}^{2}\left(v^{\alpha}\right)^{2}+\frac{1}{2}\left(c_{\alpha}^{\gamma \nu}\right)^{2} \lambda_{\gamma} \lambda_{\nu}\left(v^{\alpha}\right)^{2} \geq 0
$$

and this completes the proof.

\subsection{The evolution of the Weyl tensor.}

By means of the evolution equations found for the curvatures, we are able to write the equation satisfied by the Weyl tensor along the RB flow (1.1). In [6] the authors compute the evolution equation of the Weyl tensor during the Ricci flow (see [6, Proposition 1.1]) and we use most of their computations here.

Proposition 4.11. During the RB flow of an $n$-dimensional Riemannian manifold $(M, g)$ the Weyl tensor satisfies the following evolution equation

$$
\begin{aligned}
\frac{\partial}{\partial t} \mathrm{~W}_{i j k l}= & \Delta \mathrm{W}_{i j k l}+2\left(\mathrm{~B}(\mathrm{~W})_{i j k l}-\mathrm{B}(\mathrm{W})_{i j l k}-\mathrm{B}(\mathrm{W})_{i l j k}+\mathrm{B}(\mathrm{W})_{i k j l}\right) \\
& +2 \rho \mathrm{RW}_{i j k l}-g^{p q}\left(\mathrm{~W}_{p j k l} \mathrm{R}_{q i}+\mathrm{W}_{i p k l} \mathrm{R}_{q j}+\mathrm{W}_{i j p l} \mathrm{R}_{q k}+\mathrm{W}_{i j k p} \mathrm{R}_{q l}\right) \\
& +\frac{2}{(n-2)^{2}}\left(\operatorname{Ric}^{2} \otimes g\right)_{i j k l}+\frac{1}{(n-2)}(\operatorname{Ric} \otimes \mathrm{Ric})_{i j k l} \\
& -\frac{2 \mathrm{R}}{(n-2)^{2}}(\operatorname{Ric} \otimes g)_{i j k l}+\frac{\mathrm{R}^{2}-|\mathrm{Ric}|^{2}}{(n-1)(n-2)^{2}}(g \otimes g)_{i j k l},
\end{aligned}
$$

where $\mathrm{B}(W)_{i j k l}=g^{p q} g^{r s} \mathrm{~W}_{i p j r} \mathrm{~W}_{k q l s}$. 
Proof. By recalling the decomposition formula for the Weyl tensor (1.3) we have

$$
\begin{aligned}
\frac{\partial}{\partial t} \mathrm{~W} & =\frac{\partial}{\partial t} \operatorname{Riem}+\frac{1}{2(n-1)(n-2)}\left(\frac{\partial}{\partial t} \operatorname{R} g \otimes g+2 \frac{\partial}{\partial t} g \otimes g\right)-\frac{1}{n-2}\left(\frac{\partial}{\partial t} \operatorname{Ric} \otimes g+\operatorname{Ric} \otimes \frac{\partial}{\partial t} g\right) \\
& =\mathcal{L}_{I I}+\mathcal{L}_{0},
\end{aligned}
$$

where $\mathcal{L}_{I I}$ is the second order term in the curvatures and $\mathcal{L}$ the 0 -th one. We deal first with the higher order term; plugging in the evolution equations of Riem, Ric and R (Proposition 3.1) we get

$$
\begin{aligned}
\mathcal{L}_{I I}= & \Delta \operatorname{Riem}-\rho\left(\nabla^{2} \mathrm{R} \otimes g\right)+\frac{1-2(n-1) \rho}{2(n-1)(n-2)} \Delta \mathrm{R} g \otimes g \\
& -\frac{1}{n-2}\left(\Delta \operatorname{Ric} \otimes g-(n-2) \rho \nabla^{2} \mathrm{R} \otimes g-\rho \Delta \mathrm{R} \otimes g\right) \\
= & \Delta \operatorname{Riem}+\frac{1-2(n-1) \rho+2(n-1) \rho}{2(n-1)(n-2)} \Delta \operatorname{R} g \otimes g-\frac{1}{n-2} \Delta \operatorname{Ric} \otimes g \\
= & \Delta \mathrm{W} .
\end{aligned}
$$

Then we consider the lower order terms

$$
\begin{aligned}
\left(\mathcal{L}_{0}\right)_{i j k l}= & 2\left(B(\operatorname{Riem})_{i j k l}-B(\operatorname{Riem})_{i j l k}-B(\operatorname{Riem})_{i l j k}+B(\operatorname{Riem})_{i k j l}\right) \\
& -g^{p q}\left(\mathrm{R}_{p j k l} \mathrm{R}_{q i}+\mathrm{R}_{i p k l} \mathrm{R}_{q j}+\mathrm{R}_{i j p l} \mathrm{R}_{q k}+\mathrm{R}_{i j k p} \mathrm{R}_{q l}\right) \\
& +2 \rho \mathrm{R}\left(\mathrm{W}-\frac{1}{2(n-1)(n-2)} \operatorname{R} g \otimes g+\frac{1}{n-2} \operatorname{Ric} \otimes g\right)_{i j k l} \\
& +\frac{1}{2(n-1)(n-2)}\left(2|\operatorname{Ric}|^{2} g \otimes g-2 \rho \mathrm{R}^{2} g \oplus g-4 \operatorname{Ric} \otimes g+4 \rho \mathrm{R}^{2} g \oplus g\right)_{i j k l} \\
& -\frac{1}{n-2}\left[2(\operatorname{Riem} * \operatorname{Ric}) \otimes g-2 \operatorname{Ric}^{2} \otimes g-2 \operatorname{Ric} \otimes \operatorname{Ric}+2 \rho \operatorname{Ric} \otimes g\right]_{i j k l} \\
= & 2\left(B(\operatorname{Riem})_{i j k l}-B(\operatorname{Riem})_{i j l k}-B(\operatorname{Riem})_{i l j k}+B(\operatorname{Riem})_{i k j l}\right) \\
& -g^{p q}\left(\mathrm{R}_{p j k l} \mathrm{R}_{q i}+\mathrm{R}_{i p k l} \mathrm{R}_{q j}+\mathrm{R}_{i j p l} \mathrm{R}_{q k}+\mathrm{R}_{i j k p} \mathrm{R}_{q l}\right)+2 \rho \mathrm{RW}_{i j k l} \\
& -\frac{2}{n-2}\left[(\operatorname{Riem} * \operatorname{Ric}) \otimes g-\operatorname{Ric}^{2} \otimes g-\operatorname{Ric} \otimes \operatorname{Ric}\right]_{i j k l} \\
& -\frac{2 \mathrm{R}}{(n-1)(n-2)}(\operatorname{Ric} \otimes g)_{i j k l}+\frac{|\operatorname{Ric}|^{2}}{(n-1)(n-2)}(g \otimes g)_{i j k l},
\end{aligned}
$$

where $(\text { Riem } * \text { Ric })_{a b}=\mathrm{R}_{a p b q} \mathrm{R}_{s t} g^{p s} g^{q t}$ and $\left(\operatorname{Ric}^{2}\right)_{a b}=\mathrm{R}_{a p} \mathrm{R}_{b q} g^{p q}$.

Now we deal separately with every term containing the full curvature Riem, using its decomposition formula, expanding the Kulkarni-Nomizu products and then contracting again. We have that

$$
[(g \otimes g) * \operatorname{Ric}]_{a b}=2[\mathrm{R} g-\operatorname{Ric}]_{a b},[(\operatorname{Ric} \otimes g) * \operatorname{Ric}]_{a b}=\left[-2 \operatorname{Ric}^{2}+\operatorname{RRic}+|\operatorname{Ric}|^{2} g\right]_{a b} .
$$


Hence

$$
\begin{aligned}
(\text { Riem } * \text { Ric }) \otimes g= & (\mathrm{W} * \text { Ric }) \otimes g-\frac{2}{n-2} \operatorname{Ric}^{2} \otimes g \\
& +\frac{n \mathrm{R}}{(n-1)(n-2)} \operatorname{Ric} \otimes g+\frac{(n-1)|\mathrm{Ric}|^{2}-\mathrm{R}^{2}}{(n-1)(n-2)} g \otimes g .
\end{aligned}
$$

Then

$$
\begin{aligned}
\mathrm{R}_{q i} \mathrm{R}_{p j k l} g^{p q}= & \mathrm{R}_{q i}\left(W_{p j k l}-\frac{\mathrm{R}}{(n-1)(n-2)}\left(g_{p k} g_{j l}-g_{p l} g_{j k}\right)\right) g^{p q} \\
& +\frac{1}{n-2} \mathrm{R}_{q i}\left(\mathrm{R}_{p k} g_{j l}+\mathrm{R}_{j l} g_{p k}-\mathrm{R}_{p l} g_{j k}-\mathrm{R}_{j k} g_{p l}\right) g^{p q} \\
= & \mathrm{R}_{q i} \mathrm{~W}_{p j k l} g^{p q}-\frac{\mathrm{R}}{(n-1)(n-2)}\left(\mathrm{R}_{i k} g_{j l}-\mathrm{R}_{i l} g_{j k}\right) \\
& +\frac{1}{n-2}\left(\mathrm{R}_{i k}^{2} g_{j l}-\mathrm{R}_{i l}^{2} g_{j k}+\mathrm{R}_{i k} \mathrm{R}_{j l}-\mathrm{R}_{i l} \mathrm{R}_{j k}\right) .
\end{aligned}
$$

Interchanging the index and using the symmetry properties we get

$$
\begin{aligned}
g^{p q}\left(\mathrm{R}_{p j k l} \mathrm{R}_{q i}+\mathrm{R}_{i p k l} \mathrm{R}_{q j}+\mathrm{R}_{i j p l} \mathrm{R}_{q k}+\mathrm{R}_{i j k p} \mathrm{R}_{q l}\right) \\
\quad=g^{p q}\left(\mathrm{~W}_{p j k l} \mathrm{R}_{q i}+\mathrm{W}_{i p k l} \mathrm{R}_{q j}+\mathrm{W}_{i j p l} \mathrm{R}_{q k}+\mathrm{W}_{i j k p} \mathrm{R}_{q l}\right) \\
\quad+\frac{2}{n-2}\left(\operatorname{Ric}^{2} \otimes g\right)_{i j k l}+\frac{2}{n-2}(\operatorname{Ric} \otimes \operatorname{Ric})_{i j k l}-\frac{2 \mathrm{R}}{(n-1)(n-2)}(\operatorname{Ric} \otimes g)_{i j k l} .
\end{aligned}
$$

Finally the " $B$ "-terms:

$$
\begin{gathered}
B(\mathrm{Riem})_{a b c d}=\left(\mathrm{W}-\frac{\mathrm{R}}{2(n-1)(n-2)} g \otimes g+\frac{1}{n-2} \mathrm{Ric} \otimes g\right)_{a p b q} \\
\left(\mathrm{~W}-\frac{\mathrm{R}}{2(n-1)(n-2)} g \otimes g+\frac{1}{n-2} \operatorname{Ric} \otimes g\right)_{c s d t} g^{p s} g^{q t} \\
\left(\mathrm{~W}_{a p b q}(g \otimes g)_{c s d t}+(g \otimes g)_{a p b q} \mathrm{~W}_{c s d t}\right) g^{p s} g^{q t}=-2 \mathrm{~W}_{a d b c}-2 \mathrm{~W}_{c b d a} \\
\left(\mathrm{~W}_{a p b q}(\operatorname{Ric} \otimes g)_{c s d t}+(\operatorname{Ric} \otimes g)_{a p b q} \mathrm{~W}_{c s d t}\right) g^{p s} g^{q t}=(\mathrm{W} * \mathrm{Ric})_{a b} g_{c d}+(\mathrm{W} * \mathrm{Ric})_{c d} g_{a b} \\
-\left(\mathrm{W}_{c b d p} \mathrm{R}_{a q}+\mathrm{W}_{c p d a} \mathrm{R}_{b q}+\mathrm{W}_{a d b p} \mathrm{R}_{c q}+\mathrm{W}_{a p b d} \mathrm{R}_{d q}\right) g^{p q} \\
(g \otimes g)_{a p b d}(g \otimes g)_{c s d t} g^{p s} g^{q t}=4\left((n-2) g_{a b} g_{c d}+g_{a c} g_{b d}\right)
\end{gathered}
$$

$\left((\operatorname{Ric} \otimes g)_{a p b q}(g \otimes g)_{c s d t}+(\operatorname{Ric} \otimes g)_{c s d t}(g \otimes g)_{a p b q}\right) g^{p s} g^{q t}$

$$
=2\left((n-4) \mathrm{R}_{a b} g_{c d}+(n-4) \mathrm{R}_{c d} g_{a b}+2 \mathrm{R}_{a c} g_{b d}+2 \mathrm{R}_{b d} g_{a c}\right)
$$

$(\operatorname{Ric} \otimes g)_{a b p q}(\operatorname{Ric} \otimes g)_{c s d t} g^{p s} g^{q t}=-2 \mathrm{R}_{a b}^{2} g_{c d}-2 \mathrm{R}_{c d}^{2} g_{a b}+\mathrm{R}_{a c}^{2} g_{b d}+\mathrm{R}_{b d}^{2} g_{a c}$

$$
+(n-4) \mathrm{R}_{a b} \mathrm{R}_{c d}+2 \mathrm{R}_{a c} \mathrm{R}_{b d}+\mathrm{R}\left(\mathrm{R}_{a b} g_{c d}+\mathrm{R}_{c d} g_{a b}\right)+|\mathrm{Ric}|^{2} g_{a b} g_{c d}
$$


Now, adding the same type quantities for the different index permutations and using the symmetry properties of $\mathrm{W}$ we obtain

$$
\begin{aligned}
B(\mathrm{Riem})_{i j k l} & -B(\mathrm{Riem})_{i j l k}-B(\mathrm{Riem})_{i j j k}+B(\mathrm{Riem})_{i k j l} \\
= & B(\mathrm{~W})_{i j k l}-B(\mathrm{~W})_{i j l k}-B(\mathrm{~W})_{i l j k}+B(\mathrm{~W})_{i k j l} \\
& +\frac{1}{n-2}((\mathrm{~W} * \mathrm{Ric}) \otimes g)_{i j k l}-\frac{1}{(n-2)^{2}}\left(\mathrm{Ric}^{2} \otimes g\right)_{i j k l}+\frac{1}{2(n-2)}(\mathrm{Ric} \otimes \mathrm{Ric})_{i j k l} \\
& +\frac{\mathrm{R}}{(n-1)(n-2)^{2}}(\operatorname{Ric} \otimes g)_{i j k l}+\left(\frac{|\mathrm{Ric}|^{2}}{2(n-2)^{2}}-\frac{\mathrm{R}^{2}}{2(n-1)(n-2)^{2}}\right)(g \otimes g)_{i j k l} .
\end{aligned}
$$

We are ready to complete the computation of the 0 -th order term in the evolution equation, using the previous formulas (4.6), (4.7), (4.8)

$$
\begin{aligned}
\left(\mathcal{L}_{0}\right)_{i j k l}= & 2\left(B(\mathrm{~W})_{i j k l}-B(\mathrm{~W})_{i j l k}-B(\mathrm{~W})_{i l j k}+B(\mathrm{~W})_{i k j l}\right)+2 \rho \mathrm{RW}_{i j k l} \\
& -g^{p q}\left(\mathrm{~W}_{p j k l} \mathrm{R}_{q i}+\mathrm{W}_{i p k l} \mathrm{R}_{q j}+\mathrm{W}_{i j p l} \mathrm{R}_{q k}+\mathrm{W}_{i j k p} \mathrm{R}_{q l}\right) \\
& +\frac{2}{(n-2)^{2}}\left(\operatorname{Ric}^{2} \otimes g\right)_{i j k l}+\frac{1}{(n-2)}(\operatorname{Ric} \otimes \mathrm{Ric})_{i j k l} \\
& -\frac{2 \mathrm{R}}{(n-2)^{2}}(\operatorname{Ric} \otimes g)_{i j k l}+\frac{\mathrm{R}^{2}-|\operatorname{Ric}|^{2}}{(n-1)(n-2)^{2}}(g \oplus g)_{i j k l}
\end{aligned}
$$

\subsection{Conditions preserved in dimension three.}

In general dimension, it is very hard to find other curvature conditions preserved by the flow, and this is due principally to the complex structure of the reaction terms; for example in the evolution equation satisfied by the Ricci tensor (3.3), the reaction terms involve the full curvature tensor. Therefore it is easier to restrict our attention to the three-dimensional case, in which the Weyl part of the Riemann tensor vanishes and all the geometric informations are encoded in the Ricci tensor.

In the special case of dimension three, we can use also the evolution equation (3.10) of the pull-back of the curvature operator to obtain more refined conditions preserved, because we can rewrite the ODE associated to the evolution of $\mathcal{P}$ as a system of ODEs in the eigenvalues of $\mathcal{P}$ that, by Proposition 3.4, are nothing but the sectional curvatures of $\mathcal{R}$. This point of view has been introduced for the Ricci flow by Hamilton in [21] and can be easily generalized to the RB flow as follows.

Lemma 4.12. If $n=3$, then $\mathcal{P}_{p}$ has 3 eigenvalues $\lambda, \mu, \nu$ and the ODE fiberwise associated to equation (3.10) can be written as the following system

$$
\left\{\begin{array}{l}
\frac{d \lambda}{d t}=2 \lambda^{2}+2 \mu \nu-4 \rho \lambda(\lambda+\mu+\nu), \\
\frac{d \mu}{d t}=2 \mu^{2}+2 \lambda \nu-4 \rho \mu(\lambda+\mu+\nu), \\
\frac{d \nu}{d t}=2 \nu^{2}+2 \lambda \mu-4 \rho \nu(\lambda+\mu+\nu) .
\end{array}\right.
$$

In particular, if we assume $\lambda(0) \geq \mu(0) \geq \nu(0)$, then $\lambda(t) \geq \mu(t) \geq \nu(t)$ as long as the solution of the system exists. 
Proof. We can pointwise identify $V_{p}$ with an orthonormal frame of $\mathbb{R}^{3}$ with the standard basis. Then $\Lambda^{2} V_{p} \simeq \mathfrak{s o}(3)$ with the standard structure constants and if an algebraic operator $Q_{p}$ is diagonal, both $Q_{p}^{2}$ and $Q_{p}^{\#}$ are diagonal with respect to the same basis (for the detailed computation of this fact, see [11, Chapter 6.4]). Hence the ODE $\frac{d}{d t} Q_{p}=$ $F_{p}\left(Q_{p}\right)$ associated fiberwise to (3.10) preserves the eigenvalues of $Q_{p}$, that is, if $Q_{p}(0)$ is diagonal with respect to an orthonormal basis, $Q_{p}(t)$ stays diagonal with respect to the same basis and the ODE can be rewritten as the system (4.9) in the eigenvalues. To prove the last statement, we observe that

$$
\begin{aligned}
& \frac{d}{d t}(\lambda-\mu)=2(\lambda-\mu)((1-2 \rho)(\lambda+\mu)-(1+2 \rho) \nu) \\
& \frac{d}{d t}(\mu-\nu)=2(\mu-\nu)((1-2 \rho)(\mu+\nu)-(1+2 \rho) \lambda)
\end{aligned}
$$

Remark 4.13. We already proved that the differential operator in the evolution equation of $\mathcal{P}$ is uniformly elliptic if $\rho<1 / 2(n-1)$, that is $\rho<1 / 4$ in dimension three. Therefore any geometric condition expressed in terms of the eigenvalues is preserved along the $\mathrm{RB}$ flow if the cone identified by the condition is closed, convex and preserved by the system (4.9).

By using this method, we can prove

Proposition 4.14. Let $(M, g(t))_{t \in[0, T)}$ be a compact, three-dimensional, solution of the $R B$ flow (1.1). If $\rho<1 / 4$, then

(i) nonnegative Ricci curvature is preserved along the flow;

(ii) nonnegative sectional curvature is preserved along the flow;

(iii) the pinching inequality $\mathrm{Ric} \geq \varepsilon \mathrm{R} g$ is preserved along the flow for any $\varepsilon \leq 1 / 3$.

Proof. (i) If $\operatorname{Ric}(g(0)) \geq 0$, then $\operatorname{Ric}_{g(t)} \geq 0$.

The eigenvalues of Ric are the pairwise sums of the sectional curvatures, hence the condition is identified by the cone

$$
K_{p}=\left\{Q_{p}:(\mu+\nu)\left(Q_{p}\right) \geq 0\right\} .
$$

The closedness is obvious; in order to see that $K_{p}$ is convex, we observe that the greatest eigenvalue can be characterized by $\lambda\left(Q_{p}\right)=\max \left\{Q_{p}(v, v): v \in V_{p}|v|_{h}=1\right\}$, hence it is convex. Then the function $Q_{p} \mapsto \mu\left(Q_{p}\right)+\nu\left(Q_{p}\right)=\operatorname{tr}\left(Q_{p}\right)-\lambda\left(Q_{p}\right)$ is concave and this implies that its superlevels are convex. By system (4.9) we obtain

$$
\frac{d}{d t}(\mu+\nu)=2 \mu^{2}+2 \nu^{2}+2 \lambda(\mu+\nu)-4 \rho(\mu+\nu) \operatorname{tr}\left(Q_{p}\right) .
$$

There is the stationary solution corresponding to $\mu(0)=0=\nu(0)$. Otherwise, whenever $\mu\left(t_{0}\right)+\nu\left(t_{0}\right)=0$ with $\mu\left(t_{0}\right) \neq 0$ and $\nu\left(t_{0}\right) \neq 0, \frac{d}{d t}(\mu+\nu)\left(t_{0}\right)=2\left(\mu^{2}+\nu^{2}\right)\left(t_{0}\right)>0$, then $K$ is preserved.

(ii) If $\operatorname{Sec}(g(0)) \geq 0$, then $\operatorname{Sec}_{g(t)} \geq 0$. 
This condition is the non negativity of $\mathcal{P}$, identified by the cone $K_{p}=\left\{Q_{p}: \nu\left(Q_{p}\right) \geq 0\right\}$, which is convex as superlevel of a concave function. We suppose that $\nu\left(t_{0}\right)=0$, then

$$
\frac{d}{d t} \nu\left(t_{0}\right)=2 \lambda\left(t_{0}\right) \mu\left(t_{0}\right) \geq 0
$$

because the order between the eigenvalues is preserved and therefore $\lambda\left(t_{0}\right) \geq \mu\left(t_{0}\right) \geq 0$. (iii) For every $\varepsilon \in(0,1 / 3]$, if $\operatorname{Ric}(g(0))-\varepsilon \mathrm{R}(g(0)) g(0) \geq 0$, then $\operatorname{Ric}_{g(t)}-\varepsilon \mathrm{R}_{g(t)} g(t) \geq 0$. Translating in terms of eigenvalues of $\mathcal{P}$, the condition means $\mu\left(Q_{p}\right)+\nu\left(Q_{p}\right)-2 \varepsilon \operatorname{tr}\left(Q_{p}\right) \geq$ 0 , that is $\lambda\left(Q_{p}\right) \leq \frac{1-2 \varepsilon}{2 \varepsilon}\left(\mu\left(Q_{p}\right)+\nu\left(Q_{p}\right)\right)$, then the right cone is

$$
K_{p}=\left\{Q_{p}: \lambda\left(Q_{p}\right)-C(\varepsilon)\left(\mu\left(Q_{p}\right)+\nu\left(Q_{p}\right)\right) \leq 0\right\},
$$

where $C(\varepsilon)=\frac{1-2 \varepsilon}{2 \varepsilon} \in[1 / 2,+\infty)$. The defining function is the sum of two convex function, hence its sublevels are convex. Now, for $C=1 / 2$, that corresponds to $\varepsilon=1 / 3$, we have $\lambda(0)=\mu(0)=\nu(0)$ at each point of $M$, that is the initial metric $g(0)$ is constant sectional curvature and this condition is preserved along the flow.

For $C>1 / 2$, we suppose $\lambda\left(t_{0}\right)=C\left(\mu\left(t_{0}\right)+\nu\left(t_{0}\right)\right)$, then

$$
\begin{gathered}
\frac{d}{d t}(\lambda-C(\mu+\nu))\left(t_{0}\right)=2\left[\lambda^{2}+\mu \nu-C\left(\mu^{2}+\nu^{2}+\lambda(\mu+\nu)\right)-2 \rho \operatorname{tr}\left(Q_{p}\right)(\lambda-C(\mu+\nu))\right]\left(t_{0}\right) \\
\quad=2\left[C^{2}\left(\mu\left(t_{0}\right)+\nu\left(t_{0}\right)\right)^{2}+\mu\left(t_{0}\right) \nu\left(t_{0}\right)-C\left(\mu\left(t_{0}\right)^{2}+\nu\left(t_{0}\right)^{2}\right)-C^{2}\left(\mu\left(t_{0}\right)+\nu\left(t_{0}\right)\right)^{2}\right] \\
\leq(1-2 C)\left(\mu\left(t_{0}\right)^{2}+\nu\left(t_{0}\right)^{2}\right) \leq 0 .
\end{gathered}
$$

\subsection{Hamilton-Ivey estimate.}

A remarkable property of the three-dimensional Ricci flow is the pinching estimate, independently proved by Hamilton in [20] and Ivey in [22], which says that positive sectional curvature dominates negative sectional curvature during the Ricci flow, that is, if the initial metric $g_{0}$ has a negative sectional curvature somewhere, the Ricci flow starting at $g_{0}$ evolves the scalar curvature towards the positive semiaxis in future times, that means that there will be a greater (in absolute value) positive sectional curvature. We have generalized the pinching estimate and some consequences for positive values of the parameter $\rho$. In the same notation used before, let $\lambda \geq \mu \geq \nu$ be the ordered eigenvalues of the curvature operator.

Theorem 4.15 (Hamilton-Ivey Estimate). Let $(M, g(t))$ be a solution of the RB on a compact three-manifold such that the initial metric satisfies the normalizing assumption $\min _{p \in M} \nu_{p}(0) \geq$ -1 . If $\rho \in[0,1 / 6)$, then at any point $(p, t)$ where $\nu_{p}(t)<0$ the scalar curvature satisfies

$$
\mathrm{R} \geq|\nu|(\log (|\nu|)+\log (1+2(1-6 \rho) t)-3)
$$

Proof. We want to apply the Maximum Principle for time-dependent sets theorem 4.9, hence we need to express condition (4.10) in terms of a family of closed, convex, invariant subsets of $S^{2}\left(\Lambda^{2} V^{*}\right)$, where $(V, h(t), D(t))$ is the usual bundle isomorphism of the 
tangent bundle defined via Uhlenbeck's trick (Section 3.1). Moreover, by [10, Lemma 10.11], we already know that, for any $t \in[0, T)$, the set

$$
K_{p}(t)=\left\{\begin{array}{l}
Q_{p}: \operatorname{tr}\left(Q_{p}\right) \geq-\frac{3}{1+2(1-6 \rho) t} \text { and if } \nu\left(Q_{p}\right) \leq-\frac{1}{1+2(1-6 \rho) t} \\
\text { then } \operatorname{tr}\left(Q_{p}\right) \geq\left|\nu\left(Q_{p}\right)\right|\left(\log \left(\left|\nu\left(Q_{p}\right)\right|\right)+\log (1+2(1-6 \rho) t)-3\right)
\end{array}\right\}
$$

defines a closed invariant subset of $S^{2}\left(\Lambda^{2} V^{*}\right)$. Since, for $\rho \in[0,1 / 6), K(t)$ depends continuously on time, the space-time track of $K(t)$ is closed in $S^{2}\left(\Lambda^{2} V^{*}\right)$.

Now we show that $K_{p}(t)$ is convex for every $p \in M$ and $t \in[0, T)$. Following [11, Lemma 9.5], we consider the map

$$
\Phi: S^{2}\left(\Lambda^{2} V_{p}^{*}\right) \rightarrow \mathbb{R}^{2}, \quad \Phi\left(Q_{p}\right)=\left(\left|\nu\left(Q_{p}\right)\right|, \operatorname{tr}\left(Q_{p}\right)\right)
$$

Clearly, we have that $Q_{p} \in K_{p}(t)$ if and only if $\Phi\left(Q_{p}\right) \in A(t)$, where

$$
A(t)=\left\{\begin{array}{l}
(x, y) \in \mathbb{R}^{2}: y \geq-\frac{3}{1+2(1-6 \rho) t} ; \quad y \geq-3 x ; \\
\text { if } x \geq \frac{1}{1+2(1-6 \rho) t} \text { then } y \geq x(\log x+\log (1+2(1-6 \rho) t)-3)
\end{array}\right\}
$$

is a convex subset of $\mathbb{R}^{2}$. Then in order to show that $K_{p}(t)$ is convex is sufficient to show that the segment between any two algebraic operators in $K_{p}(t)$ is sent by the map $\Phi$ into $A(t)$.

Therefore let $Q_{p}, Q_{p}^{\prime} \in K_{p}(t), s \in[0,1]$ and $Q_{p}(s)=s Q_{p}+(1-s) Q_{p}^{\prime}$. About the first defining condition for $A(t)$, the trace is a linear functional, hence it is obviously fulfilled by $Q_{p}(s)$, while the second condition is satisfied by any algebraic operator.

The third condition is a bit tricky. If $\nu\left(Q_{p}\right), \nu\left(Q_{p}^{\prime}\right)>-\frac{1}{1+(1-6 \rho) t}$ then the condition is empty for every point of the segment because $\nu$ is a concave function. By continuity we can assume w.l.o.g. that $\nu\left(Q_{p}(s)\right) \leq-\frac{1}{1+(1-6 \rho) t}$, for every $s \in[0,1]$, hence $x\left(Q_{p}(s)\right)=$ $-\nu\left(Q_{p}(s)\right)$ is a convex function and $x\left(Q_{p}(s)\right) \leq s x\left(Q_{p}\right)+(1-s) x\left(Q_{p}^{\prime}\right)$. On the other hand the second condition implies that $x\left(Q_{p}(s)\right) \geq-y\left(Q_{p}(s)\right) / 3=-\frac{1}{3}\left(s y\left(Q_{p}\right)+(1-s) y\left(Q_{p}^{\prime}\right)\right)$. Then $\Phi\left(Q_{p}(s)\right)$ belongs to the trapezium of vertices

$$
\Phi\left(Q_{p}\right),\left(-\frac{1}{3} y\left(Q_{p}\right), y\left(Q_{p}\right)\right), \Phi\left(Q_{p}^{\prime}\right),\left(-\frac{1}{3} y\left(Q_{p}^{\prime}\right), y\left(Q_{p}^{\prime}\right)\right),
$$

contained in $A(t)$, as its vertices are and $A(t)$ is convex.

Now we prove that $K(t)$ is preserved by the system (4.9). By taking the sum of the three equations in the system (see also Remark 4.13) we get

$$
\frac{d}{d t} \operatorname{tr}\left(Q_{p}\right) \geq \frac{4}{3}(1-3 \rho) \operatorname{tr}\left(Q_{p}\right)^{2}
$$

By hypothesis, $\nu\left(Q_{p}\right)(0) \geq-1$, hence $\operatorname{tr}\left(Q_{p}\right)(0) \geq-3$ for every $p \in M$ and by integrating the previous inequality,

$$
\operatorname{tr}\left(Q_{p}\right)(t) \geq-\frac{3}{1+4(1-3 \rho) t} \geq-\frac{3}{1+2(1-6 \rho) t}
$$

which holds for any $\rho \in[0,1 / 6)$.

In order to prove that the second inequality is preserved too, we consider, for every 
$p \in M$ such that $\nu\left(Q_{p}\right)(0)<0$, the function

$$
f(t)=\frac{\operatorname{tr}\left(Q_{p}\right)}{-\nu\left(Q_{p}\right)}-\log \left(-\nu\left(Q_{p}\right)\right)-\log (1+2(1-6 \rho) t)
$$

and we compute its derivative along the flow.

$$
\begin{aligned}
\frac{d}{d t} f= & \frac{1}{\nu^{2}}\left[(-2 \nu)\left(\lambda^{2}+\mu^{2}+\nu^{2}+\lambda \mu+\lambda \nu+\mu \nu-2 \rho(\lambda+\mu+\nu)^{2}\right)\right. \\
& \left.+2(\lambda+\mu+\nu)\left(\nu^{2}+\lambda \mu-2 \rho \nu(\lambda+\mu+\nu)\right)\right] \\
& -\frac{2}{\nu}\left(\nu^{2}+\lambda \mu-2 \rho \nu(\lambda+\mu+\nu)\right)-\frac{2(1-6 \rho)}{1+2(1-6 \rho) t} \\
= & \frac{2}{\nu^{2}}\left[-\nu\left(\lambda^{2}+\mu^{2}+\lambda \mu\right)+\lambda \mu(\lambda+\mu)-\nu^{3}+2 \rho \nu^{2}(\lambda+\mu+\nu)\right]-\frac{2(1-6 \rho)}{1+2(1-6 \rho) t}
\end{aligned}
$$

As in the case of the Ricci flow, it is easy to see that the quantity $-\nu\left(\lambda^{2}+\mu^{2}+\lambda \mu\right)+$ $\lambda \mu(\lambda+\mu)$ is always nonnegative if $\nu<0$. In fact, if $\mu>0$ it is obvious, whereas if $\mu \leq 0$ one has

$$
-\nu\left(\lambda^{2}+\mu^{2}+\lambda \mu\right)+\lambda \mu(\lambda+\mu)=(\mu-\nu)\left(\lambda^{2}+\mu^{2}+\lambda \mu\right)-\mu^{3} \geq 0 .
$$

Hence we get

$$
\frac{d}{d t} f(t) \geq-2 \nu+4 \rho(\lambda+\mu+\nu)-\frac{2(1-6 \rho)}{1+2(1-6 \rho) t}
$$

If $\rho \geq 0$, since $\lambda+\mu+\nu \geq 3 \nu$, we obtain

$$
\frac{d}{d t} f \geq-2(1-6 \rho)\left(\nu+\frac{1}{1+2(1-6 \rho) t}\right) \geq 0
$$

whenever $\nu \leq-\frac{1}{1+2(1-6 \rho) t}$ and $\rho \leq 1 / 6$.

Hence, if $(\lambda, \mu, \nu)$ is a solution of system (4.9) in $[0, T)$ with $(\lambda(0), \mu(0), \nu(0)) \in K_{p}(0)$, we suppose that there is $t_{1}>0$ such that $\nu\left(t_{1}\right)<-\frac{1}{1+2(1-6 \rho) t_{1}}$. Then, either $\nu(t)<$ $-\frac{1}{1+2(1-6 \rho) t}$ for any $t \in\left[0, t_{1}\right]$, either there exists $t_{0}<t_{1}$ such that $\nu\left(t_{0}\right)=-\frac{1}{1+2(1-6 \rho) t_{0}}$ and $\nu(t)<-\frac{1}{1+2(1-6 \rho) t}$ for any $t \in\left(t_{0}, t_{1}\right]$. In the first case, by hypothesis we obtain $f(0) \geq-3$ and $\frac{d}{d t} f(t) \geq 0$ for any $t \in\left[0, t_{1}\right]$, therefore $f\left(t_{1}\right) \geq-3$; in the second case $f\left(t_{0}\right)=\frac{(\lambda+\mu+\nu)\left(t_{0}\right)}{-\nu\left(t_{0}\right)} \geq-3$ and $\frac{d}{d t} f(t) \geq 0$ for any $t \in\left[t_{0}, t_{1}\right]$, therefore again $f\left(t_{1}\right) \geq-3$, which is equivalent to the second inequality.

Remark 4.16. The extra term $4 \rho(\lambda+\mu+\nu)$ on the key-equation (4.12) requires strong assumptions on the parameter $\rho$ since we have no information on the sign of the trace. However, combining equation (4.12) with Proposition 4.4, we can enlarge the range of $\rho$ to $[0,1 / 4)$, simply by dropping the extra term, nonnegative for ancient solutions and therefore conclude that an ancient solution to the RB flow on a compact three-manifold with bounded scalar curvature has nonnegative sectional curvature for any value of $\rho \in[0,1 / 4)$ (see $[11$, Corollary 9.8]). 
Proposition 4.17. Let $(M, g(t))_{t \in(-\infty, 0]}$ be a compact, three-dimensional, ancient solution of the RB flow (1.1) with uniformly bounded scalar curvature. If $\rho \in[0,1 / 4)$ then the sectional curvature is nonnegative.

\section{Curvature estimates}

\subsection{Technical lemmas.}

Before proving the curvature estimates for the RB flow, we need some technical results. First of all, we prove the proposition:

Proposition 5.1. Let $k \in \mathbb{N}, p \in[1,+\infty]$ and $q \in[1,+\infty)$. There exists a constant $C(n, k, p, q)$ such that for all $0 \leq j \leq k$ and all tensor $T$

$$
\|T\|_{H_{j}^{r_{j}}} \leq C\|T\|_{p}^{1-\frac{j}{k}}\|T\|_{H_{k}^{q}}^{\frac{j}{k}}
$$

where $\frac{1}{r_{j}}=\frac{1-\frac{j}{k}}{p}+\frac{\frac{j}{k}}{q}$.

To prove this proposition, we need several lemmas.

Lemma 5.2. Let $p \in[1,+\infty], q \in[1,+\infty)$ and $r \in[2,+\infty)$ such that $\frac{1}{r}=\frac{1}{2 p}+\frac{1}{2 q}$. There exists a constant $C(n, r)$ such that for all tensor $T$

$$
\|\nabla T\|_{r}^{2} \leq C\|T\|_{p}\left\|\nabla^{2} T\right\|_{q} .
$$

Proof.

$$
\begin{aligned}
\|\nabla T\|_{r}^{r} & =\int_{M}\left\langle\nabla T,|\nabla T|^{r-2} \nabla T\right\rangle d \mu_{g} \\
& =-\int_{M}\left\langle T, \nabla\left(|\nabla T|^{r-2} \nabla T\right)\right\rangle d \mu_{g} \\
& =-\int_{M}\left\langle T,(r-2) \nabla^{2} T|\nabla T|^{r-3} \nabla T\right\rangle d \mu_{g}-\int_{M}\left\langle T,|\nabla T|^{r-2} \nabla^{2} T\right\rangle d \mu_{g} \\
& \leq C \int_{M}|T|\left|\nabla^{2} T \| \nabla T\right|^{r-2} d \mu_{g} \\
& \leq C\|T\|_{p}\left\|\nabla^{2} T\right\|_{q}\|\nabla T\|_{r}^{r-2},
\end{aligned}
$$

using Hölder's inequality with $\frac{r-2}{r}+\frac{1}{p}+\frac{1}{q}=1$. This ends the proof of this lemma.

Lemma 5.3. Let $k \in \mathbb{N}, p \in[1,+\infty]$ and $q \in[1,+\infty)$. There exists a constant $C(n, k, p, q))$ such that for all tensor $T$

$$
\|T\|_{H_{k+1}^{r}}^{2} \leq C\|T\|_{H_{k}^{p}}\|T\|_{H_{k+2}^{q}}
$$

where $\frac{1}{r}=\frac{1}{2 p}+\frac{1}{2 q}$.

Proof. We apply Lemma 5.2 to $\nabla^{k} T$ :

$$
\left\|\nabla^{k+1} T\right\|_{r}^{2} \leq C\left\|\nabla^{k} T\right\|_{p}\|T\|_{H_{k+2}^{q}} \leq C\|T\|_{H_{k}^{p}}\|T\|_{H_{k+2}^{q}} .
$$


On the other side, using Hölder's inequality

$$
\|T\|_{r}^{2} \leq\|T\|_{p}\|T\|_{q} \leq\|T\|_{H_{k}^{p}}\|T\|_{H_{k+2}^{q}} .
$$

Combining both inequalities gives the result.

Lemma 5.4 (Hamilton [18], Cor. 12.5). Let $k \in \mathbb{N}$. If $f:\{0, \ldots, k\} \rightarrow \mathbb{R}$ satisfies for all $0<j<k$

$$
f(j) \leq C f(j-1)^{\frac{1}{2}} f(j+1)^{\frac{1}{2}},
$$

where $C$ is a positive constant, then for all $0 \leq j \leq k$

$$
f(j) \leq C^{j(k-j)} f(0)^{1-\frac{j}{k}} f(k)^{\frac{j}{k}} .
$$

Proof of Proposition 5.1. We apply Lemma 5.4 with $f(j)=\|T\|_{H_{j}^{r_{j}}}$. Since $\frac{1}{r_{j}}=\frac{1}{2 r_{j-1}}+\frac{1}{2 r_{j+1}}$, Lemma 5.3 shows that there exists $C(n, k, p, q)$ such that

$$
f(j) \leq C f(j-1)^{\frac{1}{2}} f(j+1)^{\frac{1}{2}},
$$

and then Lemma 5.4 gives Proposition 5.1.

Lemma 5.5. For all tensors of the form $S * T$, there exists $C$ depending on the dimension and the coefficients in the expression such that

$$
|S * T| \leq C|S||T| .
$$

Proof. By Cauchy-Schwarz inequality, $\left(g^{\alpha \beta} T_{\alpha \beta}\right)^{2} \leq n T_{\alpha \beta} T^{\alpha \beta}$. Then

$$
|S * T| \leq C(n)\left|S \otimes T \otimes g^{\otimes j} \otimes\left(g^{-1}\right)^{\otimes k} \leq C(n) n^{\frac{j+k}{2}}\right| S|| T \mid .
$$

Let $k \in \mathbb{N}$, and set, for a tensor $T, F_{g}(T)=\sum_{r+s=k, r, s \geq 0} \nabla^{r} T * \nabla^{s} T * \nabla^{k} T$.

Lemma 5.6. Let $k \in \mathbb{N}$. Let $p \in[2,+\infty]$ and $q \in[2,+\infty)$ such that $\frac{1}{p}+\frac{2}{q}=1$. There exists $C(n, k, p, q, F)$ such that for all tensor $T$,

$$
\int_{M}\left|F_{g}(T)\right| d \mu_{g} \leq C\|T\|_{p}\|T\|_{H_{k}^{q}}^{2}
$$

Proof. Let us consider one term in $F_{g}(T)$ that can be written $\nabla^{r} T * \nabla^{s} T * \nabla^{k} T, r, s \geq 0$. Using Lemma 5.5 and Hölder's inequality we have

$$
\begin{aligned}
\int_{M}\left|\nabla^{r} T * \nabla^{s} T * \nabla^{k} T\right| d \mu_{g} & \leq C^{\prime} \int_{M}\left|\nabla^{r} T \| \nabla^{s} T\right|\left|\nabla^{k} T\right| d \mu_{g} \\
& \leq C^{\prime}\left\|\nabla^{r} T\right\|_{r_{1}}\left\|\nabla^{s} T\right\|_{r_{2}}\left\|\nabla^{k} T\right\|_{q} \\
& \leq C^{\prime}\|T\|_{H_{r}^{r_{1}}}\|T\|_{H_{s}^{r_{2}}}\|T\|_{H_{k}^{q}}
\end{aligned}
$$

where $\frac{1}{r_{i}}=\frac{1-\frac{r}{k}}{p}+\frac{\frac{r}{k}}{q}$. Clearly $\frac{1}{r_{1}}+\frac{1}{r_{2}}=1$. Using Lemma 5.2 we get

$$
\int_{M}\left|\nabla^{r} T * \nabla^{s} T * \nabla^{k} T\right| d \mu_{g} \leq C\|T\|_{p}\|T\|_{H_{k}^{q}}^{2} .
$$


The result follows since $F_{g}(T)$ is a linear combination of such terms.

\subsection{Curvature estimates.}

In this section we prove the following theorem:

Theorem 5.7. Assume $\rho<\frac{1}{2(n-1)}$. If $g(t)$ is a compact solution of the RB flow for $t \in[0, T)$ such that

$$
\sup _{(x, t) \in M \times[0, T)}|\operatorname{Riem}(x, t)| \leq K,
$$

then for all $k \in \mathbb{N}$ there exists a constant $C(n, \rho, k, K, T)$ such that for all $t \in(0, T]$

$$
\left\|\nabla^{k} \operatorname{Riem}_{g_{t}}\right\|_{2}^{2} \leq \frac{C}{t^{\frac{1}{2}}} \sup _{t \in[0, T)} \int_{M}\left|\operatorname{Riem}_{g_{t}}\right|^{2} d \mu_{g} .
$$

Proof. We prove the theorem by induction on $k$. A direct computation gives

$$
\begin{aligned}
\frac{\partial}{\partial t} \mid \text { Riem }\left.\right|^{2} & =\Delta\left(\mid \text { Riem }\left.\right|^{2}\right)-2 \mid \nabla \text { Riem }\left.\right|^{2}-8 \rho \mathrm{R}_{i j} \nabla^{i} \nabla^{j} \mathrm{R}+\text { Riem } * \text { Riem } * \text { Riem } \\
\frac{\partial}{\partial t} \mathrm{R}^{2} & =(1-2(n-1) \rho) \Delta\left(\mathrm{R}^{2}\right)-2(1-2(n-1) \rho)|\nabla \mathrm{R}|^{2}+4 \mathrm{R}|\mathrm{Ric}|^{2}-4 \rho \mathrm{R}^{3} .
\end{aligned}
$$

It follows that

$$
\begin{aligned}
\frac{\partial}{\partial t} \int_{M}|\operatorname{Riem}|^{2} d \mu_{g}= & -2 \int_{M}|\nabla \operatorname{Riem}|^{2} d \mu_{g}-8 \rho \int_{M} \mathrm{R}_{i j} \nabla^{i} \nabla^{j} \mathrm{R} d \mu_{g} \\
& +\int_{M} \operatorname{Riem} * \text { Riem } * \text { Riem } d \mu_{g} \\
\frac{\partial}{\partial t} \int_{M} \mathrm{R}^{2} d \mu_{g}= & -2(1-2(n-1) \rho) \int_{M}|\nabla \mathrm{R}|^{2} d \mu_{g}+\int_{M} \operatorname{Riem} * \text { Riem } * \text { Riem } d \mu_{g} .
\end{aligned}
$$

Now we want to compute $\int_{M} \mathrm{R}_{i j} \nabla^{i} \nabla^{j} \mathrm{R} d \mu_{g}$. Using Bianchi identity we have:

$$
\int_{M} \mathrm{R}_{i j} \nabla^{i} \nabla^{j} \mathrm{R} d \mu_{g}=-\frac{1}{2} \int_{M}|\nabla \mathrm{R}|^{2} d \mu_{g}
$$

We conclude that

$$
\begin{aligned}
\frac{\partial}{\partial t} \int_{M}|\operatorname{Riem}|^{2} d \mu_{g}= & -2 \int_{M} \mid \nabla \text { Riem }\left.\right|^{2} d \mu_{g}+4 \rho \int_{M}|\nabla \mathrm{R}|^{2} d \mu_{g} \\
& +\int_{M} \operatorname{Riem} * \text { Riem } * \text { Riem } d \mu_{g} \\
\frac{\partial}{\partial t} \int_{M} \mathrm{R}^{2} d \mu_{g}= & -2(1-2(n-1) \rho) \int_{M}|\nabla \mathrm{R}|^{2} d \mu_{g}+\int_{M} \operatorname{Riem} * \text { Riem } * \text { Riem } d \mu_{g} .
\end{aligned}
$$

Consider

$$
\mathcal{A}_{k}:=\int_{M}\left|\nabla^{k} \mathrm{Riem}\right|^{2} d \mu_{g}+\frac{4|\rho|}{(1-2(n-1) \rho)} \int_{M}\left|\nabla^{k} \mathrm{R}\right|^{2} d \mu_{g}
$$


and set $\beta:=\min (1,1-2(n-1) \rho)$. We have by a direct computation:

$$
\begin{aligned}
\frac{\partial}{\partial t} \mathcal{A}_{0}+\beta \mathcal{A}_{1}= & (-2+\beta) \int_{M}|\nabla \operatorname{Riem}|^{2} d \mu_{g}+\left(4 \rho-8|\rho|+\frac{4|\rho| \beta}{(1-2(n-1) \rho)}\right) \int_{M}|\nabla \mathrm{R}|^{2} d \mu_{g} \\
& +\int_{M} \operatorname{Riem} * \operatorname{Riem} * \operatorname{Riem} d \mu_{g} .
\end{aligned}
$$

Note that $-2+\beta \leq 0$ and $4 \rho-8|\rho|+\frac{4|\rho| \beta}{(1-2(n-1) \rho)} \leq 0$. Using the assumption on the boundedness of $\mid$ Riem $\mid$ we have:

$$
\frac{\partial}{\partial t} \mathcal{A}_{0}+\beta \mathcal{A}_{1} \leq C\|\operatorname{Riem}\|_{2}^{2}
$$

Consider $f_{1}(t):=\mathcal{A}_{0}+\beta t \mathcal{A}_{1}$. We have

$$
f_{1}^{\prime}(t)=\mathcal{A}_{0}^{\prime}+\beta \mathcal{A}_{1}+\beta t \mathcal{A}_{1}^{\prime} \leq C\|\operatorname{Riem}\|_{2}^{2}+C \beta t\|\operatorname{Riem}\|_{2}^{2} \leq C(1+t)\|\operatorname{Riem}\|_{2}^{2} .
$$

It follows, integrating this inequality

$$
\int_{M}|\nabla \operatorname{Riem}|^{2} d \mu_{g} \leq \mathcal{A}_{1} \leq \frac{1}{\beta t} f_{1}(t) \leq \frac{C}{t}\|\operatorname{Riem}\|_{2}^{2}
$$

This is what we wanted to prove for $k=1$.

More generally, we want to control all the derivatives of the curvature. Assume that the conclusion of the theorem is true up to $(k-1)$. As we did before, a straightforward computation gives:

$$
\begin{aligned}
\frac{\partial}{\partial t} \int_{M}\left|\nabla^{k} \mathrm{Riem}\right|^{2} d \mu_{g}= & -2 \int_{M}\left|\nabla^{k+1} \operatorname{Riem}\right|^{2} d \mu_{g}+4 \rho \int_{M}\left|\nabla^{k+1} \mathrm{R}\right|^{2} d \mu_{g} \\
& +\sum_{r+s=k, r, s \geq 0} \int_{M} \nabla^{r} \operatorname{Riem} * \nabla^{s} \operatorname{Riem} * \nabla^{k} \operatorname{Riem} d \mu_{g} \\
\frac{\partial}{\partial t} \int_{M}\left|\nabla^{k} \mathrm{R}\right|^{2} d \mu_{g}= & -2(1-2(n-1) \rho) \int_{M}\left|\nabla^{k+1} \mathrm{R}\right|^{2} d \mu_{g} \\
& +\sum_{r+s=k, r, s \geq 0} \int_{M} \nabla^{r} \operatorname{Riem} * \nabla^{s} \operatorname{Riem} * \nabla^{k} \operatorname{Riem} d \mu_{g} .
\end{aligned}
$$

We need to estimate $\sum_{r+s=k, r, s \geq 0} \int_{M} \nabla^{r}$ Riem $* \nabla^{s}$ Riem $* \nabla^{k}$ Riem $d \mu_{g}$. For this we use Lemma 5.6 with $p=+\infty$ and $q=2$ :

$$
\sum_{r+s=k, r, s \geq 0} \int_{M} \nabla^{r} \operatorname{Riem} * \nabla^{s} \operatorname{Riem} * \nabla^{k} \operatorname{Riem} d \mu_{g} \leq C\|\operatorname{Riem}\|_{\infty}\|\operatorname{Riem}\|_{H_{k}^{2}}^{2} .
$$


Using Lemma 5.2 we get

$$
\begin{aligned}
\sum_{r+s=k, r, s \geq 0} \int_{M} \nabla^{r} \operatorname{Riem} * \nabla^{s} \operatorname{Riem} * \nabla^{k} \operatorname{Riem} d \mu_{g} \leq & C \| \text { Riem }\left\|_{\infty}\right\| \nabla^{k} \text { Riem } \|_{2}^{2} \\
& +C \| \text { Riem }\left\|_{\infty}\right\| \text { Riem } \|_{2}^{2} \\
\leq & C \| \text { Riem }\left\|_{\infty}\right\| \text { Riem }\left\|_{2}\right\| \nabla^{k+1} k \text { Riem } \|_{2} \\
& +C \| \text { Riem }\left\|_{\infty}\right\| \text { Riem } \|_{2}^{2} \\
\leq & \frac{1}{2} \| \nabla^{k+1} k \text { Riem } \|_{2}^{2} \\
& +C\left(\| \text { Riem }\left\|_{\infty}^{2}+\right\| \text { Riem } \|_{\infty}\right) \| \text { Riem } \|_{2}^{2} .
\end{aligned}
$$

Similarly as before, and using this last estimate, we have

$$
\frac{\partial}{\partial t} \mathcal{A}_{k}+\beta \mathcal{A}_{k+1} \leq C\|\operatorname{Riem}\|_{2}^{2}
$$

Now define $f_{k}(t):=\sum_{j=0}^{k} \frac{\beta^{j} t^{j}}{j !} \mathcal{A}_{j}$. We have

$$
\begin{aligned}
f_{k}^{\prime}(t) & =\sum_{j=0}^{k-1} \frac{\beta^{j} t^{j}}{j !}\left(\mathcal{A}_{j}^{\prime}+\beta \mathcal{A}_{j+1}\right)+\frac{\beta^{k} t^{k}}{k !} \mathcal{A}_{k}^{\prime} \\
& \leq C\|\operatorname{Riem}\|_{2}^{2} \sum_{j=0}^{k} \frac{\beta^{j} t^{j}}{j !} \leq C(1+t)^{k} \| \text { Riem } \|_{2}^{2} .
\end{aligned}
$$

Now, integrating this inequality,

$$
\int_{M}\left|\nabla^{k} \operatorname{Riem}\right|^{2} d \mu_{g} \leq \mathcal{A}_{k} \leq \frac{k !}{\beta^{k} t^{k}} f_{k}(t) \leq \frac{C}{t^{k}} \| \text { Riem } \|_{2}^{2},
$$

and this ends the proof of the theorem.

\subsection{Long time existence.}

In this section we will prove the following result.

Theorem 5.8. Assume $\rho<\frac{1}{2(n-1)}$. If $g(t)$ is a compact solution of the RB flow on a maximal time interval $[0, T), T<+\infty$, then

$$
\limsup _{t \rightarrow T} \max _{M}|\operatorname{Riem}(\cdot, t)|=+\infty .
$$

Proof. If the Riemann tensor is uniformly bounded as $t \rightarrow T$, by using the interpolation inequalities in Lemma 5.3, from Theorem 5.7 we immediately get the estimates

$$
\int_{M}\left|\nabla^{k} \operatorname{Riem}\right|^{p} d \mu_{g} \leq C_{k, p}
$$


for all $k \in \mathbb{N}$ and $p<+\infty$. Now, let $E_{k}:=\mid \nabla^{k}$ Riem $\left.\right|^{2}$. Then, for all $p<+\infty$ we have

$$
\int_{M}\left(\left|E_{k}\right|^{p}+\left|\nabla E_{k}\right|^{p}\right) d \mu_{g} \leq C_{k, p}^{\prime} \text {. }
$$

Thus, by Sobolev inequality, if $p>k$, one has

$$
\max _{M}\left|E_{k}\right|^{p} \leq C_{t} \int_{M}\left(\left|E_{k}\right|^{p}+\left|\nabla E_{k}\right|^{p}\right) d \mu_{g}
$$

Notice that the constant $C_{t}$ depends on the metric $g(t)$, but it does not depend on the derivatives of $g(t)$. Moreover, from [18, Lemma 14.2], it follows that the metrics are all equivalent. Hence, the constant $C_{t}$ is uniformly bounded as $t \rightarrow T$ and, from the previous estimates, it follows that, if $|\operatorname{Riem}| \leq C$ on $M \times[0, T)]$, for every $k \in \mathbb{N}$ one has

$$
\max _{M} \mid \nabla^{k} \text { Riem } \mid \leq C_{k},
$$

where the constant $C_{k}$ depends only on the initial value of the metric and the constant C.

Arguing now as in [18, Section 14], it follows that the metrics $g(t)$ converge to some limit metric $g(T)$ in the $C^{\infty}$ topology (with all their time/space ordinary partial derivatives, once written in local coordinates), hence, we can restart the flow with this initial metric $g(T)$, obtaining a smooth flow in some larger time interval $[0, T+\delta)$, in contradiction with the fact that $T$ was the maximal time of smooth existence. This completes the proof of Theorem 5.8.

\section{THE SOLITONS}

We pass now to the analysis of gradient $\rho$-Einstein solitons. All the results of this section can be found in [8] and [9]. We say that a gradient $\rho$-Einstein soliton is a Riemannian manifold $(M, g), n \geq 3$, endowed with a smooth function $f: M \rightarrow \mathbb{R}$, such that the metric $g$ satisfies the equation

$$
\text { Ric }+\nabla^{2} f=\rho \mathrm{R} g+\lambda g,
$$

for some constants $\rho, \lambda \in \mathbb{R}, \rho \neq 0$. The soliton is trivial whenever $\nabla f$ is parallel. As usual, the $\rho$-Einstein soliton is steady for $\lambda=0$, shrinking for $\lambda>0$ and expanding for $\lambda<0$. The function $f$ is called a $\rho$-Einstein potential of the gradient $\rho$-Einstein soliton.

Corresponding to special values of the parameter $\rho$, we refer to the $\rho$-Einstein solitons with different names, according to the Riemannian tensor which rules the flow. Hence, for $\rho=1 / 2$ we will have Einstein solitons, for $\rho=1 / n$ traceless Ricci solitons and for $\rho=1 / 2(n-1)$ Schouten solitons.

In this section we will collect some facts concerning gradient $\rho$-Einstein solitons. First of all, it is easy to show that gradient $\rho$-Einstein solitons give rise to solutions to the RB flow (1.1).

Theorem 6.1 ([9]). If $\left(M, g_{0}, f_{0}\right)$ is a complete gradient $\rho$-Einstein soliton with Einstein constant $\lambda$, then there exist

i. a family of metrics $g(t)$, solution to the RB flow (1.1), with $g(0)=g_{0}$, 
ii. a family of diffeomorphisms $\phi(t): M \rightarrow M$, with $\phi(0)=\mathrm{id}_{M}$,

iii. a family of functions $f(t): M \rightarrow \mathbb{R}$ with $f(0)=f_{0}$,

all three defined for every $t$ such that $\tau(t):=-2 \lambda+1>0$. These families have the following properties:

1. the family $\phi(t)$ is generated by the vector-fields

$$
X(t)(x)=\frac{1}{\tau(t)}\left(\nabla^{g_{0}} f_{0}\right)(\phi(t)(x))
$$

i.e.

$$
\frac{\partial}{\partial t} \phi(t)(x)=\frac{1}{\tau(t)}\left(\nabla^{g_{0}} f_{0}\right)(\phi(t)(x)),
$$

2. the metric $g(t)$ is given by pullback through $\phi$ and rescaling, i.e.

$$
g(t)=\tau(t) \phi(t)^{*} g_{0}
$$

3. the function $f(t)$ is given as well by pullback, i.e.

$$
f(t)=f_{0} \circ \phi(t) .
$$

Proof. We set $\tau(t)=-2 \lambda t+1$. As $\nabla^{g_{0}} f_{0}$ is a complete vector-field, there exists a 1parameter family of diffeomorphisms $\phi(t, \cdot): M \rightarrow M$ generated by the time dependent family of vector fields $X(t, \cdot):=\frac{1}{\tau(t)} \nabla^{g_{0}} f_{0}(\phi(t, \cdot))$, for every $t$ such that $\tau(t)>0$. We also set $f(t, \cdot)=\left(f_{0} \circ \phi\right)(t, \cdot)$ and $g(t)=\tau(t) \phi(t)^{*} g_{0}$. We compute

$$
\frac{\partial}{\partial t} g(t)=-\frac{2 \lambda}{\tau(t)} g(t)+\tau(t) \frac{\partial}{\partial t} \phi(t, \cdot)^{*} g_{0} \cdot
$$

By the definition of the Lie derivative, we have that $\frac{\partial}{\partial t} \phi(t, \cdot)^{*} g_{0}=\mathscr{L}_{(\phi(t)-1) * \frac{\partial}{\partial t} \phi(t, \cdot)} \phi(t, \cdot)^{*} g_{0}$. On the other hand, equation (6.3) implies that

$$
\frac{\partial \phi}{\partial t}(t, \cdot)=\frac{1}{\tau(t)}\left(\nabla^{g_{0}} f_{0}\right)(\cdot)=\frac{1}{\tau(t)} \phi(t, \cdot)_{*} \nabla^{g(t)} f(t, \cdot),
$$

where we used the fact that $\phi(t, \cdot)^{*} \nabla^{g_{0}} f_{0}=\nabla^{\phi(t, \cdot)^{*} g_{0}} \phi(t, \cdot)^{*} f_{0}=\nabla^{g(t)} f(t, \cdot)$. Combining these two facts, we have that

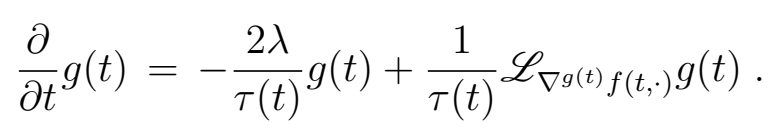

Having this at hand, we compute

$$
\begin{aligned}
-\operatorname{Ric}_{g(t)} & =\phi(t, \cdot)^{*}\left(-\operatorname{Ric}\left(g_{0}\right)\right)=\phi(t, \cdot)^{*}\left(\frac{1}{2} \mathscr{L}_{\nabla^{g_{0} f_{0}}} g_{0}-\lambda g_{0}-\rho \mathrm{R}\left(g_{0}\right) g_{0}\right) \\
& =\frac{1}{2}\left(\frac{1}{\tau(t)} \mathscr{L}_{\nabla^{g(t)} f(t, \cdot)} g(t)-\frac{2}{\tau(t)} \lambda g(t)\right)-\frac{\rho}{\tau(t)} \mathrm{R}\left(\tau(t)^{-1} g(t)\right) g(t) \\
& =\frac{1}{2} \frac{\partial}{\partial t} g(t)-\frac{\rho}{\tau(t)} \mathrm{R}\left(\tau(t)^{-1} g(t)\right) g(t)
\end{aligned}
$$


and we observe that $\mathrm{R}\left(\tau(t)^{-1} g(t)\right)=\tau(t) \mathrm{R}_{g(t)}$. In other words, we have obtained

$$
\frac{\partial}{\partial t} g(t)=-2\left[\operatorname{Ric}_{g(t)}-\rho \mathrm{R}_{g(t)} g(t)\right]
$$

and the proof is complete.

Concerning the regularity of this structures we have the following result.

Theorem 6.2 ([9]). A gradient $\rho-$ Einstein soliton is real analytic, provided $\rho \neq 1 / n$.

We say that a smooth function $f: M \rightarrow \mathbb{R}$ is rectifiable in an open set $U \subset M$ if and only if $\left|\nabla f_{\mid U}\right|$ is constant along every regular connected component of the level sets of $f_{\mid U}$. In particular, it can be seen that $f_{\mid U}$ only depends on the signed distance $r$ to the regular connected component of some of its level sets. If $U=M$, we simply say that $f$ is rectifiable. Consequently, a gradient soliton is called rectifiable if and only if it admits a rectifiable potential function. The rectifiability turns out to be one of main property of the $\rho$-Einstein solitons, as we will show in the following theorem.

Theorem 6.3 ([8]). Every gradient $\rho$-Einstein soliton is rectifiable.

Moreover it can be proved that, locally, the solitons can be foliated by hypersurfaces with constant mean curvature and constant induced scalar curvature, about a regular connected component of a level set of the potential $f$. It is worth noticing that Theorem 6.3 fails to be true in the case of gradient Ricci solitons. In fact, even though all of the easiest nontrivial examples - such as the Gaussian soliton and the round cylinder in the shrinking case, or the Hamilton's cigar (also known in the physics literature as Witten's black hole) and the Bryant soliton in the steady case - are rectifiable, it is easy to check, for instance, that the Riemannian product of rectifiable steady gradient Ricci solitons gives rise to a new steady soliton, which is generically not rectifiable.

Finally, we list here some classification results. First of all in the compact case we have the following

Theorem 6.4 ([8]). Let $(M, g), n \geq 3$, be compact gradient $\rho$-Einstein soliton. Then, the following cases occur.

(i) If $\rho \leq 1 / 2(n-1)$, then either $\lambda>0$ and $\mathrm{R}>0$ or the soliton is trivial.

(i-bis) If $\rho=1 / 2(n-1)$, then the soliton is trivial.

(ii) If $1 / 2(n-1)<\rho<1 / n$, then either $\lambda<0$ and $\mathrm{R}<0$ or the soliton is trivial.

(iii) If $1 / n \leq \rho$, the soliton is trivial.

In particular, for solitons corresponding to these special values of $\rho$, we get

Corollary 6.5. Every compact gradient Einstein, Schouten or traceless Ricci soliton is trivial.

In the general (also noncompact) case the following results were proved.

Theorem 6.6 ([8]). Let $(M, g)$ be a three-dimensional gradient $\rho$-Einstein soliton with $\rho<0$ and $\lambda \leq 0$ or $\rho \geq 1 / 2$ and $\lambda \geq 0$. If $(M, g)$ has positive sectional curvature, then it is rotationally symmetric. 
Theorem $6.7([8])$. Let $(M, g)$ be a complete $n$-dimensional, $n \geq 4$, locally conformally flat gradient $\rho$-Einstein soliton with $\rho<0$ and $\lambda \leq 0$ or $\rho \geq 1 / 2$ and $\lambda \geq 0$. If $(M, g)$ has positive sectional curvature, then it is rotationally symmetric.

We say that a Riemannian manifold is rigid if, for some $k \in\{0, \ldots,(n-1)\}$, its universal cover, endowed with the lifted metric and the lifted potential function, is isometric to the Riemannian product $N^{k} \times \mathbb{R}^{n-k}$, where $N^{k}$ is a $k$-dimensional Einstein manifold and $f=\frac{\lambda}{2}|x|^{2}$ on the Euclidean factor. We also recall that $g$ has nonnegative radial sectional curvature if $\operatorname{Riem}(E, \nabla f, E, \nabla f) \geq 0$ for every vector field $E$ orthogonal to $\nabla f$.

Theorem 6.8 ([9]). Let $(M, g)$ be a complete, noncompact, gradient shrinking $\rho$-Einstein soliton with $0<\rho \leq 1 / 2(n-1)$. If $g$ has bounded curvature, nonnegative radial sectional curvature, and nonnegative Ricci curvature, then $(M, g)$ is rigid.

In particular, every complete, noncompact, gradient shrinking $\rho$-Einstein soliton with $0<\rho \leq 1 / 2(n-1)$ and nonnegative sectional curvature is rigid.

Among all the $\rho$-Einstein solitons, a class of particular interest is given by gradient Schouten solitons, namely Riemannian manifolds satisfying

$$
\text { Ric }+\nabla^{2} f=\frac{\mathrm{R}}{2(n-1)} g+\lambda g,
$$

for some smooth function $f$ and some constant $\lambda \in \mathbb{R}$. In the steady case, we can prove the following triviality result, which holds true in every dimension without any curvature assumption.

Theorem 6.9 ([8]). Every complete gradient steady Schouten soliton is trivial, hence Ricci flat.

In particular, every complete three-dimensional gradient steady Schouten soliton is isometric to a quotient of $\mathbb{R}^{3}$. In analogy with Perelman's classification of threedimensional gradient shrinking Ricci solitons [25], subsequently proved without any curvature assumption in [5], we have the following theorem.

Theorem 6.10 ([8]). Let $(M, g)$ be a complete three-dimensional gradient shrinking Schouten soliton. Then, it is isometric to a finite quotient of either $\mathbb{S}^{3}$, or $\mathbb{R}^{3}$ or $\mathbb{R} \times \mathbb{S}^{2}$.

\section{REFERENCES}

1. T. Aubin, Métriques riemanniennes et courbure, J. Diff. Geom. 4 (1970), 383-519.

2. A. L. Besse, Einstein manifolds, Springer-Verlag, Berlin, 2008.

3. J.-P. Bourguignon, Ricci curvature and Einstein metrics, Global differential geometry and global analysis (Berlin, 1979), Lecture Notes in Math., vol. 838, Springer, Berlin, 1981, pp. 42-63.

4. S. Brendle, Convergence of the Yamabe flow for arbitrary initial energy, J. Diff. Geom. 69 (2005), no. 2, 217-278.

5. H.-D. Cao, B.-L. Chen, and X.-P. Zhu, Recent developments on Hamilton's Ricci flow, Surveys in differential geometry. Vol. XII. Geometric flows, vol. 12, Int. Press, Somerville, MA, 2008, pp. 47-112.

6. G. Catino and C. Mantegazza, Evolution of the Weyl tensor under the Ricci flow, Ann. Inst. Fourier (2011), 1407-1435. 
7. G. Catino, P. Mastrolia, D. D. Monticelli, and M. Rigoli, Conformal Ricci solitons and related integrability conditions, ArXiv Preprint Server - http://arxiv.org, to appear on Adv. Geom., 2014.

8. G. Catino and L. Mazzieri, Gradient Einstein solitons, ArXiv Preprint Server - http://arxiv.org, 2012.

9. G. Catino, L. Mazzieri, and S. Mongodi, Rigidity of gradient Einstein shrinkers, ArXiv Preprint Server http://arxiv.org, to appear on Comm. Cont. Math., 2013.

10. B. Chow, S.-C. Chu, D. Glickenstein, C. Guenther, J. Isenberg, T. Ivey, D. Knopf, P. Lu, F. Luo, and L. Ni, The Ricci flow: techniques and applications. Part II. Analytic aspects, Mathematical Surveys and Monographs, vol. 144, American Mathematical Society, Providence, RI, 2008.

11. B. Chow and D. Knopf, The Ricci flow: an introduction, Mathematical Surveys and Monographs, vol. 110, American Mathematical Society, Providence, RI, 2004.

12. E. Delay, Inversion d'opérateurs de courbures au voisinage de la métrique euclidienne, ArXiv Preprint Server - http://arxiv.org, 2014.

13. D. M. DeTurck, Deforming metrics in the direction of their Ricci tensors, J. Diff. Geom. 18 (1983), no. 1, 157-162.

14. __ Deforming metrics in the direction of their Ricci tensors (improved version), Collected Papers on Ricci Flow (H.-D. Cao, B. Chow, S.-C. Chu, and S.-T. Yau, eds.), Series in Geometry and Topology, vol. 37, Int. Press, 2003, pp. 163-165.

15. J. Jr. Eells and J. H. Sampson, Harmonic mappings of Riemannian manifolds, Amer. J. Math. 86 (1964), 109-160.

16. A. E. Fischer, An introduction to conformal Ricci flow, ArXiv Preprint Server - http://arxiv.org, 2003.

17. S. Gallot, D. Hulin, and J. Lafontaine, Riemannian geometry, Springer-Verlag, 1990.

18. R. S. Hamilton, Three-manifolds with positive Ricci curvature, J. Diff. Geom. 17 (1982), no. 2, 255-306.

19. ___ Four-manifolds with positive curvature operator, J. Diff. Geom. 24 (1986), no. 2, 153-179.

20. The formation of singularities in the Ricci flow, Surveys in differential geometry, Vol. II (Cambridge, MA, 1993), Int. Press, Cambridge, MA, 1995, pp. 7-136.

21. __ Four-manifolds with positive isotropic curvature, Comm. Anal. Geom. 5 (1997), no. 1, 1-92.

22. T. Ivey, Ricci solitons on compact three-manifolds, Differential Geom. Appl. 3 (1993), no. 4, 301-307.

23. P. Lu, J. Qing, and Y. Zheng, A note on conformal Ricci flow, ArXiv Preprint Server - http://arxiv.org, 2011.

24. C. Mantegazza, Lecture notes on mean curvature flow, Progress in Mathematics, vol. 290, Birkhäuser/Springer Basel AG, Basel, 2011.

25. G. Perelman, The entropy formula for the Ricci flow and its geometric applications, ArXiv Preprint Server http://arxiv.org, 2002.

26. A. Savas-Halilaj and K. Smoczyk, Bernstein theorems for length and area decreasing minimal maps, Calc.Var. and Partial Differential Equations. 50 (2014), 549-577.

27. H. Schwetlick and M. Struwe, Convergence of the Yamabe flow for "large" energies, J. Reine Angew. Math. 562 (2003), 59-100.

28. P. Topping, Lectures on the Ricci flow, London Mathematical Society Lecture Note Series, vol. 325, Cambridge University Press, Cambridge, 2006.

29. H. F. Weinberger, Invariant sets for weakly coupled parabolic and elliptic systems, Rend. Mat. (6) 8 (1975), 295-310, Collection of articles dedicated to Mauro Picone on the occasion of his ninetieth birthday.

30. R. Ye, Global existence and convergence of Yamabe flow, J. Diff. Geom. 39 (1994), no. 1, 35-50. 
(Giovanni Catino) Dipartimento di Matematica, Politecnico di Milano, Piazza Leonardo DA VinCi 32, Milano, ITALY, 20133

E-mail address, G. Catino: giovanni.catino@polimi.it

(Laura Cremaschi) Scuola Normale Superiore di Pisa, Piazza dei Cavalieri 7, Pisa, Italy, 56126

E-mail address, Laura Cremaschi: Laura.cremaschiesns. it

(Zindine Djadli) Institut Fourier, 100 Rue des Maths, BP 74, St. Martin D'Heres, France, 38402 and Laboratorio Fibonacci, PiazZa dei CaValieri 7, Pisa, Italy, 56126

E-mail address, Z. Djadli: Zindine.Djadli@ujf-grenoble.fr

(Carlo Mantegazza) Dipartimento di Matematica, Università di Napoli "Federico II", Via Cintia, Monte S. ANGelo, NAPOli, ITALY, I-80126

E-mail address, C. Mantegazza: c . mantegazzaesns . it

(Lorenzo Mazzieri) Scuola Normale Superiore di Pisa, Piazza dei CAVAlieri 7, Pisa, Italy, 56126

E-mail address, L. Mazzieri: 1.mazzieri@sns. it 